\title{
Numerical Computation of Normal Forms around some Periodic Orbits of the Restricted Three Body Problem
}

\author{
Àngel Jorba ${ }^{(1)}$ and Jordi Villanueva ${ }^{(2)}$
}

April 7, 1997

(1) Departament de Matemàtica Aplicada i Anàlisi, Universitat de Barcelona, Gran Via 585, 08007 Barcelona, Spain. E-mail: angel@maia.ub.es

(2) Departament de Matemàtica Aplicada I, Universitat Politècnica de Catalunya, Diagonal 647, 08028 Barcelona, Spain. E-mail: jordi@tere.upc.es

\begin{abstract}
In this paper we introduce a general methodology for computing (numerically) the normal form around a periodic orbit of an autonomous analytic Hamiltonian system. The process follows two steps. First, we expand the Hamiltonian in suitable coordinates around the orbit and second, we perform a standard normal form scheme, based on the Lie series method. This scheme is carried out up to some finite order and, neglecting the remainder, we obtain an accurate description of the dynamics in a (small enough) neighbourhood of the orbit. In particular, we obtain the invariant tori that generalize the elliptic directions of the periodic orbit. On the other hand, bounding the remainder one obtains lower estimates for the diffusion time around the orbit.

This procedure is applied to an elliptic periodic orbit of the spatial Restricted Three Body Problem. The selected orbit belongs to the Lyapunov family associated to the vertical oscillation of the equilibrium point $L_{5}$. The mass parameter $\mu$ has been chosen such that $L_{5}$ is unstable but the periodic orbit is still stable. This allows to show the existence of regions of effective stability near $L_{5}$ for values of $\mu$ bigger that the Routh critical value. The computations have been done using formal expansions with numerical coefficients.
\end{abstract}




\section{Contents}

1 Introduction $\quad 3$

2 Methodology 5

2.1 Adapted coordinates . . . . . . . . . . . . . . . . 6

2.2 Bounds on the domain of definition of the adapted coordinates . . . . . . 8

2.3 Floquet transformation . . . . . . . . . . . . . . . . . . 9

2.3.1 The variational flow ................... 10

2.3.2 The change of variables . . . . . . . . . . . . . 10

2.3.3 The reduced Hamiltonian . . . . . . . . . . . . . . 12

2.4 Complexification of the Hamiltonian . . . . . . . . . . . . . . 12

2.5 Computing the normal form . . . . . . . . . . . . . . . 13

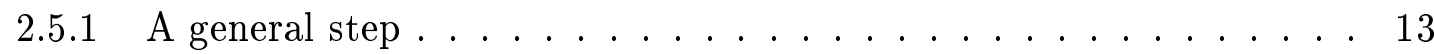

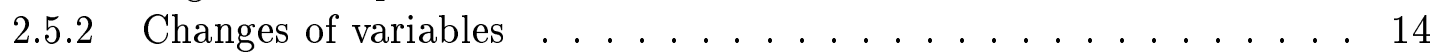

2.6 The normal form . . . . . . . . . . . . . . . . . 15

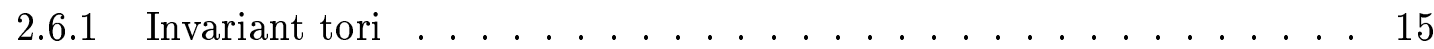

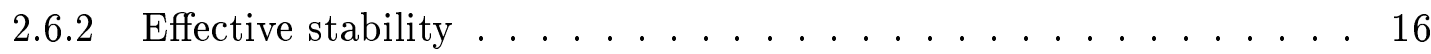

2.7 Bounds on the domain of convergence of the normal form . . . . . . . . 16

2.8 Bounds on the diffusion speed . . . . . . . . . . . . . . 17

3 Application to the spatial RTBP $\quad \mathbf{2 1}$

3.1 The Restricted Three Body Problem . . . . . . . . . . . . . . . . 21

3.2 The vertical family of periodic orbits of $L_{5} \ldots \ldots \ldots \ldots$. . . . . . 22

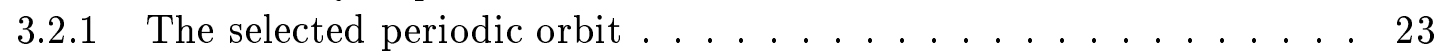

3.3 Expansion of the Hamiltonian of the RTBP . . . . . . . . . . . 23

3.4 Bounds on the norm of the Hamiltonian . . . . . . . . . . . . . 24

3.5 Numerical implementation . . . . . . . . . . . . . . . 25

3.6 Results in a concrete example . . . . . . . . . . . . . . . 27

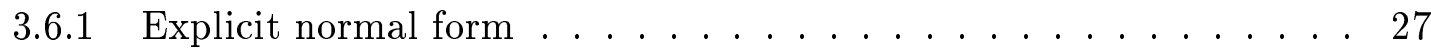

3.6.2 Effective stability estimates . . . . . . . . . . . . 29

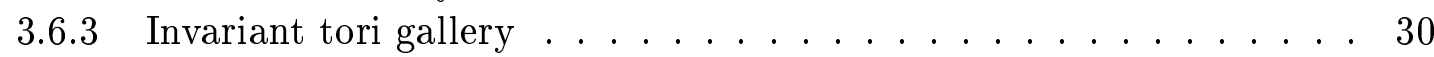

3.7 Software . . . . . . . . . . . . . . . . . . 31

4 Proof of Proposition 1

$\begin{array}{lll}5 & \text { Acknowledgements } & 37\end{array}$

$\begin{array}{ll}\text { References } & \mathbf{3 7}\end{array}$ 


\section{Introduction}

Normal forms are a standard tool in Hamiltonian mechanics to study the dynamics in a neighbourhood of invariant objects, like equilibrium points, periodic orbits or invariant tori. Usually, these normal forms are obtained as divergent series, but their asymptotic character is what makes them useful. From a theoretical point of view, they provide nonlinear approximations to the dynamics in a neigbourhood of the invariant object, that allows to obtain information about the real solutions of the system by taking the normal form up to a suitable finite order. In several cases, the remainder turns out to be exponentially small with respect to some parameters (see [20], [17], [26], [6] and [14]). This is the basis to derive the classical Nekhoroshev estimates (see [18], [15], [22], [5] and [19]). Moreover, although those series are usually divergent on open sets, it is still possible in some cases to prove convergence on special sets with empty interior (Cantor-like sets). This is the basis of KAM theory (see [1], [21] and [5]).

From a more practical point of view, normal forms can be used as a computational method to obtain very accurate approximations to the dynamics in a neighbourhood of the selected invariant object. They have been applied, for instance, to compute invariant manifols (see [11] and [27]), invariant tori (see [10], [28] and [13]) or to produce estimates on the diffusion time near linearly stable invariant objects (see [8], [25] and [12]). This numerical approach is the one taken along this paper, that can be considered a numerical application of [14]. Before the formulation of the methodology, let us mention some related results that can be found in the literature.

Let us consider an analytic Hamiltonian $H$ with $\ell$ degrees of freedom, having an elliptic equilibrium point at the origin. Under generic conditions of nonresonance and nondegeneracy, KAM theory ensures that there is plenty of $\ell$-dimensional invariant tori around the point. If $\ell$ is 2 the point is nonlinearly stable (the 2-dimensional tori split the 3 -dimensional energy levels $H=h$ in two connected components), but in the general case

$\ell>2$ it is widely accepted that some diffusion can take place. In this case, the use of normal forms allows to produce lower bounds on this diffusion time, that are exponentially big with the distance to the origin. This gives rise to the so-called effective stability, that is, even in the cases when the system is not stable it looks like it were (i.e., the time needed to observe the unstability is very long, usually longer than the expected lifetime of the studied physical system).

The stability of the Trojan asteroids is a classical example of this kind. A first model for this problem is provided by the Restricted Three Body Problem (RTBP), where the problem boils down to estimate the speed of diffusion around an elliptic equilibrium point of a 3 degrees of freedom autonomous Hamiltonian system. In order to produce good estimates, it is necessary to compute numerically the normal form around the point, up to some finite order (see [25], and also [4] for a slightly different approach). This allows to derive much better estimates than the ones obtained by only using purely theoretical methods. This has also been extended to consider time-dependent periodic perturbations, in a very natural way: in a first step one computes the periodic orbit that replaces the equilibrium point and, by means of a translation, one puts it at the origin. Now, a single linear (and periodic with respect to the time) change of variables removes the time dependence at first order, and then, the methodology above can be extended without major problems (see [10], [12] and [28]).

In this paper we will focus on the problem of computing the normal form around 
a linearly stable periodic orbit of an autonomous analytic Hamiltonian system with 3 degrees of freedom. One can think that the problem can be solved in the same way we have mentioned for periodically perturbed Hamiltonian systems, that is, to bring the orbit at the origin and to apply a Floquet transformation. The main difficulty of this method comes from the following fact: due to the symplectic structure of the problem, the monodromy matrix around the periodic orbit has, at least, two eigenvalues equal to 1. This implies that the reduced Floquet matrix is going to have two zero eigenvalues, and this does not allow to continue with the normal form process.

For this reason, here we have taken a different approach. As we are going to work around a non-degenerate elliptic periodic orbit, we expect that the monodromy matrix has the following structure (may be after a linear change of variables): a 2-dimensional Jordan box with two eigenvalues 1 , plus two couples of conjugate eigenvalues of modulus 1, all different. Under some generical conditions of non-resonance and non-degeneracy, we have that: a) the Jordan box spans, in the complete system, a one parametric family of periodic orbits, b) each couple of conjugate eigenvalues spans a Cantorian family of 2-dimensional invariant tori, and c) if we consider the excitation coming from both elliptic directions, we obtain a Cantorian family of 3-dimensional invariant tori (see [7], [3], [14] and [23] for the proofs of these facts). Hence, we will use suitable coordinates for this structure: we will introduce an angular variable $(\theta)$ as coordinate along the initial orbit, and a symplectically conjugate action variable $(I)$. For the normal directions we will simply apply the procedure used for the examples mentioned above: we will translate the orbit to the origin and we will perform a complex Floquet change to remove the dependence on the angle of the normal variational equations, and to put them in diagonal form (by means of a complex change of coordinates). Denoting by $\omega_{0}$ the frequency of the selected periodic orbit and by $\omega_{1,2}$ the two normal frequencies, the Hamiltonian will take the form

$$
H(\theta, q, I, p)=\omega_{0} I+i \omega_{1} q_{1} p_{1}+i \omega_{2} q_{2} p_{2}+\cdots,
$$

with the periodic orbit given by $I=0, q=p=0$. This is suitable to start the normal form process.

These ideas have been applied to a concrete example coming from the Restricted Three Body Problem. The selected periodic orbit belongs to the Lyapunov family associated to the vertical oscillation of the equilibrium point $L_{5}$. The mass parameter $\mu$ is chosen big enough such that $L_{5}$ is unstable, but not too big in order to have the selected orbit normally elliptic (see Section 3.2). The first changes of variables are computed taking advantage of the particularities of this concrete model, but they can be extended to similar problems. The normal form is then computed by a standard recurrent procedure (based on Lie series) up to order 16. From the normal form we can easily obtain (approximate) periodic orbits (belonging to the previously mentioned Lyapunov family) as well as invariant tori of dimensions 2 and 3 that, as has been mentioned before, generalize the linear oscillations around the orbit. Moreover, bounding the remainder of this approximate normal form allows to derive bounds on the diffusion time around the orbit.

The computations have been done using formal expansions for the involved series, but with numerical coefficients. The algebraic manipulators needed have been written from scratch by the authors, using $\mathrm{C}$.

To end this section, we comment how this paper is organized. In Section 2 we present a general (and formal) formulation of the normal form methodology, that is directly adapted to the case of a linearly stable periodic orbit of a Hamiltonian system with three degrees of 
freedom, but that can be used (slighty modified) in some other different contexts (as the study of systems with more than three degrees of freedom, or around periodic orbits with some hyperbolic directions). This formulation has as a reference point the objective to obtain bounds for the diffusion speed around the orbit. Section 3 contains the application to the RTBP.

\section{Methodology}

We consider a real analytic Hamiltonian system with three degrees of freedom given by

$$
H \equiv H\left(X, Y, Z, P_{X}, P_{Y}, P_{Z}\right)
$$

where $X, Y$ and $Z$ are the positions and $P_{X}, P_{Y}$ and $P_{Z}$ the conjugate momenta. Let $(f(\theta), g(\theta))$ be a $2 \pi$-periodic parametrization of an elliptic periodic orbit of the system, with period $\frac{2 \pi}{\omega_{0}}$. Here, $f=\left(f_{1}, f_{2}, f_{3}\right)$ and $g=\left(g_{1}, g_{2}, g_{3}\right)$ are real analytic functions with the normalization $f_{3}(0)=0$. Now we will assume the following condition (that will be satisfied by the selected example):

Condition $\mathbf{C}$ : the projection of the orbit into the coordinates $\left(Z, P_{Z}\right)$ is a simple curve close to a circle.

Note that this may not be directly satisfied by a generic example. The reason we have use it is that it simplifies the computations. In cases when it is not satisfied one should try to introduce changes of variables in order to obtain such condition. For instance, this is always possible if we are dealing with Lyapunov orbits not too far from the equilibrium point. Condition $\mathbf{C}$ implies that one can write

$$
f_{3}(\theta)=A \sin (\theta)+\hat{f}_{3}(\theta), \quad g_{3}(\theta)=A \cos (\theta)+\hat{g}_{3}(\theta),
$$

where $\left|\hat{f}_{3}(\theta)\right|$ and $\left|\hat{g}_{3}(\theta)\right|$ are small on the set $|\operatorname{Im}(\theta)| \leq \rho$, for some $\rho>0$ (this will be stated rigorously in Section 2.2). Moreover, without loss of generality, we can assume $A>0$. Then, the function

$$
\Delta(\theta)=\left(f_{3}^{\prime}(\theta)\right)^{2}+\left(g_{3}^{\prime}(\theta)\right)^{2}
$$

is always positive and "close" to the non-zero constant $A^{2}$. The non-vanishing character of $\Delta$ is necessary for technical reasons. It is used in Section 2.1 to define the (canonical) transformation (16). Thus, condition $\mathbf{C}$ is needed to guarantee the diffeomorphic character of this transformation.

Before continuing with the formal description of the methodology, let us give some notation to be used in the next sections. As it has been mentioned before, we will introduce a new set of variables $(\theta, q, I, p)$ to describe a neighbourhood of the periodic orbit. For functions depending on these variables, we will use the following notations.

If $f(\theta, q, I, p)$ is an analytic function, we expand it as

$$
f(\theta, q, I, p)=\sum_{k, l, m} f_{k, l, m}(\theta) I^{k} q^{l} p^{m}
$$

being $f_{k, l, m}(\theta)$ an analytic $2 \pi$-periodic function, that can also be expanded as

$$
f_{k, l, m}(\theta)=\sum_{s} f_{k, l, m, s} \exp (i s \theta)
$$


where $i=\sqrt{-1}$. In those sums, the indices $k, l, m$ and $s$ range on $\mathbb{N}, \mathbb{N}^{2}, \mathbb{N}^{2}$ and $\mathbb{Z}$ respectively. We also introduce the following definition of degree for a monomial $f_{k, l, m}(\theta) I^{k} q^{l} p^{m}$, to be used along the paper:

$$
\operatorname{deg}\left(I^{k} q^{l} p^{m}\right)=2 k+|l|_{1}+|m|_{1},
$$

where $|a|_{1}=\sum_{j}\left|a_{j}\right|$. The reason for counting twice the degree of $I$ will be clearer in Section 2.5.

Let us now assume that $f(\theta, q, I, p)$ is defined on the complex domain

$$
\mathcal{D}(\rho, R)=\left\{(\theta, q, I, p) \in \mathbb{C}^{6}:|\operatorname{Im}(\theta)| \leq \rho,|I| \leq R_{0},\left|q_{j}\right| \leq R_{j},\left|p_{j}\right| \leq R_{j+2}, j=1,2\right\},
$$

where $R=\left(R_{0}, R_{1}, R_{2}, R_{3}, R_{4}\right)$. Then, we introduce the norms

$$
\left\|f_{k, l, m}\right\|_{\rho}=\sup _{|\operatorname{Im}(\theta)| \leq \delta}\left|f_{k, l, m}(\theta)\right|,
$$

and

$$
\|f\|_{\rho, R}=\sup _{\mathcal{D}(\rho, R)}|f(\theta, q, I, p)| .
$$

We note that the explicit computation of these norms for a given function can be difficult, but they can be bounded by the following norms,

$$
\left|f_{k, l, m}\right|_{\rho}=\sum_{s}\left|f_{k, l, m, s}\right| \exp (|s| \rho)
$$

and

$$
|f|_{\rho, R}=\sum_{k, l, m}\left|f_{k, l, m}\right|_{\rho} R_{0}^{k} R_{1}^{l_{1}} R_{2}^{l_{2}} R_{3}^{m_{1}} R_{4}^{m_{2}},
$$

that are easier to control. Moreover, using standard Cauchy estimates on $f$, we have for the coefficients of the expansion (4) that,

$$
\left\|f_{k, l, m}\right\|_{\rho} \leq \frac{\|f\|_{\rho, R}}{R_{0}^{k} R_{1}^{l_{1}} R_{2}^{l_{2}} R_{3}^{m_{1}} R_{4}^{m_{2}}}
$$

and, if $0<\rho_{0}<\rho$, that

$$
\left\|\frac{\partial f_{k, l, m}}{\partial \theta}\right\|_{\rho-\rho_{0}} \leq \frac{\left\|f_{k, l, m}\right\|_{\rho}}{\rho_{0}}
$$

\subsection{Adapted coordinates}

The initial system of (Cartesian) coordinates $\left(X, Y, Z, P_{X}, P_{Y}, P_{Z}\right)$ is not a suitable system of reference to describe the dynamics around the periodic orbit. As it has been mentioned in the Introduction, the natural system of reference should contain an angular variable describing the orbit. Hence, we want to replace the coordinates of (1) by a new system of canonical coordinates $(\theta, q, I, p)=\left(\theta, q_{1}, q_{2}, I, p_{1}, p_{2}\right)$, with a real analytic transformation, depending on $\theta$ in a $2 \pi$-periodic way. The change has to satisfy that the periodic orbit corresponds to the set $q=p=0$ and $I=0$.

To construct this change, we take advantage on the hypothesis that $\Delta$ is different from zero. To give this transformation explicitly, let us start by defining the function

$$
\sigma(\theta)=\frac{g_{3}^{\prime}(\theta) f_{3}^{\prime \prime}(\theta)-g_{3}^{\prime \prime}(\theta) f_{3}^{\prime}(\theta)}{\Delta(\theta)^{2}}
$$


which is small if condition $\mathbf{C}$ holds. Let $\alpha(\theta, s)$ be the only solution of

$$
s=\alpha(\theta, s)+\frac{1}{2} \sigma(\theta) \alpha(\theta, s)^{2}
$$

such that $\alpha(\theta, 0)=0$. Then, if we denote by $F$ the function

$$
F(q, \theta, p, I)=I+\sum_{j=1}^{2}\left(g_{j}^{\prime}(\theta) q_{j}-f_{j}^{\prime}(\theta) p_{j}\right)
$$

the change is given by

$$
\begin{aligned}
X & =f_{1}(\theta)+q_{1}, & P_{X} & =g_{1}(\theta)+p_{1}, \\
Y & =f_{2}(\theta)+q_{2}, & P_{Y} & =g_{2}(\theta)+p_{2}, \\
Z & =f_{3}(\theta)-\frac{g_{3}^{\prime}(\theta)}{\Delta(\theta)} \alpha(\theta, F(q, \theta, p, I)), & P_{Z} & =g_{3}(\theta)+\frac{f_{3}^{\prime}(\theta)}{\Delta(\theta)} \alpha(\theta, F(q, \theta, p, I)) .
\end{aligned}
$$

Now we are going to prove that this is a canonical transformation and, in Section 2.2, we will show that if $\left|\hat{f}_{3}\right|$ and $\left|\hat{g}_{3}\right|$ (see (2)) are small enough, then (16) is a diffeomorphism from a complex domain in the variables $(\theta, q, I, p)$ to a (complex) neighbourhood of the periodic orbit.

Lemma 1 The transformation (16) is symplectic.

Proof: Let us consider the (formal) generating function $S\left(\theta, q_{1}, q_{2}, P_{X}, P_{Y}, P_{Z}\right)$ given by

$$
\begin{aligned}
S= & S_{0}(\theta)+\left(f_{1}(\theta)+q_{1}\right)\left(P_{X}-g_{1}(\theta)\right)+\left(f_{2}(\theta)+q_{2}\right)\left(P_{Y}-g_{2}(\theta)\right)+ \\
& +S_{1}(\theta)\left(P_{Z}-g_{3}(\theta)\right)+S_{2}(\theta)\left(P_{Z}-g_{3}(\theta)\right)^{2}
\end{aligned}
$$

with

$$
\begin{aligned}
S_{0}^{\prime}(\theta) & =f_{1}(\theta) g_{1}^{\prime}(\theta)+f_{2}(\theta) g_{2}^{\prime}(\theta)+f_{3}(\theta) g_{3}^{\prime}(\theta) \\
S_{1}(\theta) & =f_{3}(\theta) \\
S_{2}(\theta) & =-\frac{g_{3}^{\prime}(\theta)}{2 f_{3}^{\prime}(\theta)}
\end{aligned}
$$

Let us see that (16) is obtained from the relations

$$
I=\frac{\partial S}{\partial \theta}, \quad p_{1}=\frac{\partial S}{\partial q_{1}}, \quad p_{2}=\frac{\partial S}{\partial q_{2}}, \quad X=\frac{\partial S}{\partial P_{X}}, \quad Y=\frac{\partial S}{\partial P_{Y}}, \quad Z=\frac{\partial S}{\partial P_{Z}} .
$$

From $p_{1}=\frac{\partial S}{\partial q_{1}}, p_{2}=\frac{\partial S}{\partial q_{2}}, X=\frac{\partial S}{\partial P_{X}}$ and $Y=\frac{\partial S}{\partial P_{Y}}$, we easily obtain the expressions for $X$, $Y, P_{X}$ and $P_{Y}$ in (16). Moreover, putting $I=\frac{\partial S}{\partial \theta}$ in (15) one obtains

$$
F(q, \theta, p, I)=f_{3}^{\prime}\left(P_{Z}-g_{3}\right)-\frac{g_{3}^{\prime \prime} f_{3}^{\prime}-g_{3}^{\prime} f_{3}^{\prime \prime}}{2\left(f_{3}^{\prime}\right)^{2}}\left(P_{Z}-g_{3}\right)^{2}+\frac{\left(g_{3}^{\prime}\right)^{2}}{f_{3}^{\prime}}\left(P_{Z}-g_{3}\right)
$$

and from $Z=\frac{\partial S}{\partial P_{Z}}$ we derive

$$
f_{3}^{\prime}\left(Z-f_{3}\right)=-g_{3}^{\prime}\left(P_{Z}-g_{3}\right)
$$


Then, from the last two equations it is not difficult to obtain the expressions of the change for $Z$ and $P_{Z}$.

In principle, this is only a formal construction (note that $S_{2}(\theta)$ has singularities), but we remark that the transformation (16) is well defined around the whole periodic orbit. A more rigorous (and much more tedious) verification, without any singularity, can be obtained by directly checking the symplectic character of the differential of the change.

For further uses, let us denote by $\mathcal{F}(q, p, \theta, I)$ the transformation

$$
\mathcal{F}:(q, p, \theta, I) \rightarrow\left(X, Y, Z, P_{X}, P_{Y}, P_{Z}\right)
$$

\subsection{Bounds on the domain of definition of the adapted coordi- nates}

Now we are going to give conditions to ensure the diffeomorphic character of the canonical transformation (16) in a neighbourhood of the periodic orbit. These conditions have to be explicit enough to be applied in a practical example. More concretely, what we want to show is that if $\hat{f}_{3}$ and $\hat{g}_{3}$ are small enough, then the transformation $\mathcal{F}$ of (17) is invertible from a complex neighbourhood of $\operatorname{Im}(\theta)=0, I=0, q=0$ and $p=0$, to a complex neighbourhood of the periodic orbit.

The main difficulty is the presence of the angular variable $\theta$. It turns the study of the injectivity of (16) into a "global" problem (we want injectivity around the whole periodic orbit), instead of the classical local formulation (injectivity around a fixed point).

To check this global injectivity, we will use the following construction. First, we note that for a fixed $\theta$ the correspondence $(q, p) \rightarrow\left(X, Y, P_{X}, P_{Y}\right)$ is clearly bijective. Hence, to check the diffeomorphic character of $\mathcal{F}$, what we have to prove is that the correspondence $(\theta, F) \rightarrow\left(Z, P_{Z}\right)$ is also injective. For this purpose, we consider an auxiliar transformation of the form

$$
(\theta, \beta) \rightarrow(x, y), \quad x=f(\theta)-g^{\prime}(\theta) \beta, \quad y=g(\theta)+f^{\prime}(\theta) \beta,
$$

with $f(\theta)=A \sin (\theta)+\hat{f}(\theta)$ and $g(\theta)=A \cos (\theta)+\hat{g}(\theta)$, where $A$ is a positive number, and $\hat{f}, \hat{g}$ are arbitrary real analytic functions, $2 \pi$-periodic on $\theta$. We note that replacing $\frac{\alpha}{\Delta}$ by $\beta$ in the expressions of $Z$ and $P_{Z}$, we have that the correspondence $(\theta, \beta) \rightarrow\left(Z, P_{Z}\right)$ is analogous to (18). We will show that, if $\hat{f}$ and $\hat{g}$ are small (enough) functions, and we consider values of $\theta$ in a complex neighbourhod of $\mathbb{T}^{1}$ and of $\beta$ in a complex neighbourhod of $\beta=0$, both small enough, then (18) is injective. This result is contained in the next proposition.

Proposition 1 With the notations given above, we consider fixed values of $0<R_{\beta}<1 / 2$ and $0<\rho<1$. Assuming the $|.|_{\rho}$ norms (see (10)) of $\hat{f}, \hat{g}, \hat{f}^{\prime}, \hat{g}^{\prime}, \hat{f}^{\prime \prime}$ and $\hat{g}^{\prime \prime}$ small enough (condition depending only on $A$ and $R_{\beta}$ ), then there exist positive values $\delta_{0}\left(R_{\beta}, \rho\right)$ and $\delta_{1}\left(R_{\beta}, \rho\right)$, such that, if $\delta_{0}>\delta_{1}$, then the transformation (18) is injective on $|\operatorname{Im} \theta| \leq \rho$ and $|\beta| \leq R_{\beta}$.

The proof of this proposition is contained in Section 4.

Remark 1 Admissible values of $\delta_{0}$ and $\delta_{1}$ are explicitly constructed during the proof of the proposition. They verify that $\delta_{1} \rightarrow 0$ and that $\delta_{0}$ is bounded away from zero, when the |. I $_{\mathrm{o}}$ norms displayed above go to zero. 
Remark 2 Of course, Proposition 1 is which motivates condition $\mathbf{C}$ on the initial periodic orbit. Without this assumption, to invert (18) can be very difficult.

Then, assuming $\hat{f}_{3}$ and $\hat{g}_{3}$ small enough, we deduce from this result that the correspondence $(\theta, \beta) \rightarrow\left(Z, P_{Z}\right)$ is injective if $|\operatorname{Im}(\theta)| \leq \rho$ and $|\beta| \leq R_{\beta}$, for some small $\rho>0$ and $R_{\beta}>0$.

The next step is to invert the correspondence $(\theta,.) \rightarrow \alpha(\theta,$.$) of (14)$. We note that $\alpha$ is explicitly given by

$$
\alpha(\theta, s)=\sum_{k \geq 1} 2^{k}\left(\begin{array}{c}
1 / 2 \\
k
\end{array}\right)(\sigma(\theta) s)^{k-1} s,
$$

and hence, it is well defined if $(\theta, s)$ verifies $|s|<\frac{1}{2|\sigma(\theta)|}$. Moreover, if we take a fixed $\theta$, and values $s_{0} \neq s_{1}$ with the previous restriction, we deduce from (14) that $\alpha\left(\theta, s_{0}\right) \neq \alpha\left(\theta, s_{1}\right)$. As we are also interested in the size of $\alpha$, we remark that if $|s|<\frac{1}{2|\sigma(\theta)|}$, then we can write $|\alpha(\theta, s)| \leq \frac{1}{|\sigma(\theta)|}\left(1-(1-2|s||\sigma(\theta)|)^{1 / 2}\right)$. This bound is an increasing function of $|\sigma(\theta)|$ and $|s|$.

Finally, from all these remarks, we deduce that if we consider values for $(\theta, q, I, p)$ belonging to $\mathcal{D}(\rho, R)$, with all the components of the vector $R$ small enough such that

$$
|F(q, \theta, p, I)|<\sup _{|\operatorname{Im}(\theta)| \leq \rho}\left\{\frac{1}{2|\sigma(\theta)|}\right\}
$$

and

$$
\frac{|\alpha(\theta, F(q, \theta, p, I))|}{|\Delta(\theta)|} \leq R_{\beta}
$$

then, we can guarantee that the transformation $\mathcal{F}$ of (17) is a diffeomorphism from this domain to a neighbourhood of the periodic orbit.

\subsection{Floquet transformation}

If we rewrite the Hamiltonian (1) in the adapted coordinates (16), it takes the form:

$$
H(\theta, q, I, p)=h_{0}+\omega_{0} I+\frac{1}{2}\left(q^{\top}, p^{\top}\right) A(\theta)\left(\begin{array}{c}
q \\
p
\end{array}\right)+\sum_{j \geq 3} H_{j}(\theta, q, I, p),
$$

where we keep, for simplicity, the name $H$ for the transformed Hamiltonian. Here, $h_{0}$ is the energy level of the periodic orbit, $A(\theta)$ is a symmetric matrix ( $2 \pi$-periodic on $\theta$ ) and the terms $H_{j}$ are homogeneous polynomials of degree $j$ (see (6)). The next step is to remove the angular dependence of $\mathrm{A}$ on $\theta$, that is, to reduce the normal variational equations of the orbit to constant coefficients. So, we will perform a canonical change of variables, linear with respect to $(q, p)$ and depending $2 \pi$-periodically on $\theta$, such that it reduces $A$ to constant coefficients (this is, a Floquet transformation). As the initial Hamiltonian is real, we would like to use a real Floquet transformation. This is not possible in general (it is well known that one can be forced to double the period to obtain a real change) but it can be done in some particular situations. In the case we are considering (reducibility around a periodic orbit of a Hamiltonian system), the change can be selected to be real if, for instance, the projection of the monodromy matrix associated to the orbit into their normal directions diagonalizes without any negative eigenvalue. Note that this holds on any elliptic periodic orbit under the assumption of different normal eigenvalues. 


\subsubsection{The variational flow}

Let $\Psi(t)$ be the variational matrix, $\Psi(0)=I d_{6}$, of the periodic orbit for the initial Hamiltonian system (1). Then, $\Phi(t)=\left(D \mathcal{F}\left(0,0, \omega_{0} t, 0\right)\right)^{-1} \Psi(t) D \mathcal{F}(0,0,0,0)$ is the variational matrix of the orbit for the Hamiltonian system (21), that is, for the system expressed in the variables $(q, p, \theta, I)$. We note that the variational equations in these variables are given by:

$$
\dot{\Phi}=\left(\begin{array}{cccc}
J_{4} A & 0 & \partial_{p I}^{2} H \\
\partial_{I q}^{2} H & \partial_{I p}^{2} H & 0 & -\partial_{q I}^{2} H \\
0 & 0 & 0 & 0
\end{array}\right) \Phi
$$

where the matrix of this linear system is evaluated on the periodic orbit, $\theta(t)=\omega_{0} t, I(t)=$ 0 and $p(t)=q(t)=0$. Here, $\partial_{u v}^{2} H$ denotes the matrix of partial derivatives $\left(\frac{\partial^{2} H}{\partial u_{j} \partial v_{k}}\right)_{j, k}$, and $J_{4}$ is the matrix of the canonical 2-form of $\mathbb{C}^{4}$. Let $\widetilde{\Phi}(t)$ be the 4-dimensional matrix obtained by taking the first 4 rows and colums of $\Phi(t)$ (it corresponds to the variational flow in the normal directions of the periodic orbit). If we use the notation $\Phi=\frac{\partial(q, p, \theta, I)}{\partial\left(q^{0}, p^{0}, \theta^{0}, I^{0}\right)}$, we can identify $\widetilde{\Phi}=\frac{\partial(q, p)}{\partial\left(q^{0}, p^{0}\right)}$. Then, we obtain from (22) that

$$
\dot{\tilde{\Phi}}=J_{4} A\left(\omega_{0} t\right) \widetilde{\Phi}+\left.\left(\begin{array}{c}
\partial_{p I}^{2} H \\
-\partial_{q I}^{2} H
\end{array}\right)\right|_{\left(\omega_{0} t, 0,0,0\right)} \frac{\partial I}{\partial\left(q^{0}, p^{0}\right)} .
$$

Moreover, from the last row of the matrix of $(22)$, we have that $\frac{d}{d t}\left(\frac{\partial I}{\partial\left(q^{0}, p^{0}\right)}\right)=0$, and hence, from the initial conditions for $\Phi$ at $t=0$, we deduce that $\left(\frac{\partial I}{\partial\left(q^{0}, p^{0}\right)}\right)(t)=0$. So, the matrix $\widetilde{\Phi}$ is the solution of the linear periodic system

$$
\dot{\tilde{\Phi}}=J_{4} A\left(\omega_{0} t\right) \widetilde{\Phi}, \quad \widetilde{\Phi}(0)=I d_{4} .
$$

As we are interested in a numerical implementation of this method, we remark that it is not difficult to check that

$$
D \mathcal{F}\left(0,0, \omega_{0} t, 0\right)=\left(\begin{array}{cccccc}
1 & 0 & 0 & 0 & f_{1}^{\prime} & 0 \\
0 & 1 & 0 & 0 & f_{2}^{\prime} & 0 \\
-\frac{g_{1}^{\prime} g_{3}^{\prime}}{\Delta} & -\frac{g_{2}^{\prime} g_{3}^{\prime}}{\Delta} & \frac{f_{1}^{\prime} g_{3}^{\prime}}{\Delta} & \frac{f_{2}^{\prime} g_{3}^{\prime}}{\Delta} & f_{3}^{\prime} & -\frac{g_{3}^{\prime}}{\Delta} \\
0 & 0 & 1 & 0 & g_{1}^{\prime} & 0 \\
0 & 0 & 0 & 1 & g_{2}^{\prime} & 0 \\
\frac{g_{1}^{\prime} f_{3}^{\prime}}{\Delta} & \frac{g_{2}^{\prime} f_{3}^{\prime}}{\Delta} & -\frac{f_{1}^{\prime} f_{3}^{\prime}}{\Delta} & -\frac{f_{2}^{\prime} f_{3}^{\prime}}{\Delta} & g_{3}^{\prime} & \frac{f_{3}^{\prime}}{\Delta}
\end{array}\right),
$$

being all the components of the matrix evaluated on $\theta=\omega_{0} t$. Moreover, we also remark that, to compute the matrix $\left(D \mathcal{F}\left(0,0, \omega_{0} t, 0\right)\right)^{-1}$, we can use that it is symplectic with respect to the 2 -form $\widetilde{J}_{6}$,

$$
\widetilde{J}_{6}=\left(\begin{array}{cc}
J_{4} & 0 \\
0 & J_{2}
\end{array}\right)
$$

\subsubsection{The change of variables}

Now, let us introduce $\mathcal{C}=\widetilde{\Phi}\left(\frac{2 \pi}{\omega_{0}}\right)$, the monodromy matrix of the normal variational equations (23). From the assumed linearly stable character of the initial orbit, we have 
that $\mathcal{C}$ has four different eigenvalues of modulus 1 , that is, eigenvalues of the form $\exp \left(i \alpha_{j}\right)$ and $\exp \left(-i \alpha_{j}\right)$, for $j=1,2$, with $\alpha_{j} \in \mathbb{R}$. To compute them, we can use that (as $\mathcal{C}$ is a symplectic matrix) the characteristic polynomial of $\mathcal{C}$ takes the form $Q(\lambda)=$ $\lambda^{4}-a \lambda^{3}+b \lambda^{2}-a \lambda+1$, being $a=t r_{1}(\mathcal{C})$ (the trace of $\mathcal{C}$ ), and $b=\operatorname{tr}_{2}(\mathcal{C})$ (that is, the sum of the main minors of order 2 of $\mathcal{C})$. From these expressions, we obtain the following relations:

$$
\begin{aligned}
a & =2 \cos \left(\alpha_{1}\right)+2 \cos \left(\alpha_{2}\right), \\
b & =2+4 \cos \left(\alpha_{1}\right) \cos \left(\alpha_{2}\right) .
\end{aligned}
$$

Hence, $\cos \left(\alpha_{1}\right)$ and $\cos \left(\alpha_{2}\right)$ are the solutions for $c$ of the quadratic equation $4 c^{2}-2 a c+b-$ $2=0$. Let $w^{(j)}=u^{(j)}+i v^{(j)}$ be eigenvectors of $\mathcal{C}$ associated to the eigenvalues $\exp \left(i \alpha_{j}\right)$, for $j=1,2$. From the symplectic character of $\mathcal{C}$ with respect to $J_{4}$, we have that $w^{(1) \top} J_{4} w^{(2)}=$ $w^{(1) \top} J_{4} \bar{w}^{(2)}=0$, where the bar denotes the complex conjugation. Moreover, from the nondegenerate character of $J_{4}$, we also have that $w^{(1) \top} J_{4} \bar{w}^{(1)} \neq 0$ (in fact, this is a purely imaginary number). Hence, if we introduce $C$ the matrix that has as columns the vectors $u^{(1)}, u^{(2)}, v^{(1)}$ and $v^{(2)}$, then $C^{\top} J_{4} C$ takes the form

$$
\left(\begin{array}{cc}
0 & D \\
-D & 0
\end{array}\right)
$$

with $D=\operatorname{diag}\left(d_{1}, d_{2}\right), d_{j}=u^{(j) \top} J_{4} v^{(j)}, j=1,2$, both different from zero. We can assume $d_{j}>0$ (otherwise we only have to change $\alpha_{j}$ by $-\alpha_{j}$, which means that $v^{(j)}$ is replaced by $-v^{(j)}$, changing the sign of $d_{j}$ ). So, we can replace $u^{(j)}$ and $v^{(j)}$ by $u^{(j)} / \sqrt{d_{j}}$ and $v^{(j)} / \sqrt{d_{j}}$, and then one has that $C^{\top} J_{4} C=J_{4}$ (that is, $C$ is a symplectic matrix).

Before continuing, let us introduce the following matrices:

$$
\Omega_{1}(\theta)=\left(\begin{array}{cc}
\cos \left(\frac{\alpha_{1}}{2 \pi} \theta\right) & 0 \\
0 & \cos \left(\frac{\alpha_{2}}{2 \pi} \theta\right)
\end{array}\right), \quad \Omega_{2}(\theta)=\left(\begin{array}{cc}
\sin \left(\frac{\alpha_{1}}{2 \pi} \theta\right) & 0 \\
0 & \sin \left(\frac{\alpha_{2}}{2 \pi} \theta\right)
\end{array}\right),
$$

and

$$
\Omega=\left(\begin{array}{cc}
\Omega_{1} & \Omega_{2} \\
-\Omega_{2} & \Omega_{1}
\end{array}\right)
$$

Then, using this notation, what we have by construction is that $C^{-1} \mathcal{C} C=\Omega(2 \pi) \equiv \Omega^{*}$. From here we deduce that, taking $\mathcal{M}=C \tilde{\Omega} C^{-1}$, with

$$
\tilde{\Omega}=\left(\begin{array}{cc}
0 & \tilde{\Omega}^{*} \\
-\tilde{\Omega}^{*} & 0
\end{array}\right), \quad \tilde{\Omega}^{*}=\left(\begin{array}{cc}
\alpha_{1} & 0 \\
0 & \alpha_{2}
\end{array}\right),
$$

we have $\exp (\mathcal{M})=\mathcal{C}$. A direct verification shows, from the symplectic character of $C$, that $J_{4} \mathcal{M}$ is a symmetric matrix. Then, we have that the matrix $B(\theta)$ defined as

$$
B(\theta)=C^{-1} \exp \left(\frac{1}{2 \pi} \mathcal{M} \theta\right) \tilde{\Phi}\left(\frac{\theta}{\omega_{0}}\right)^{-1}
$$

is symplectic and $2 \pi$-periodic on $\theta$. The symplectic character of $B$ is clear, and the fact that it is $2 \pi$-periodic can be checked showing that $B$ verifies the linear differential system

$$
B^{\prime}=C^{-1} \frac{\mathcal{M}}{2 \pi} B-\frac{1}{\omega_{0}} B J_{4} A,
$$


and that by construction we have $B(0)=B(2 \pi)$. Then, we use the matrix $B$ to define the following canonical transformation,

$$
\begin{aligned}
\left(\begin{array}{l}
q \\
p
\end{array}\right) & =B(\theta)^{-1}\left(\begin{array}{l}
x \\
y
\end{array}\right) \\
I & =\xi-\frac{1}{2}\left(x^{\top}, y^{\top}\right) J_{4} B^{\prime}(\theta) B(\theta)^{-1}\left(\begin{array}{l}
x \\
y
\end{array}\right) .
\end{aligned}
$$

The canonical character of (25) is equivalent to the following equalities: if we put $\zeta^{\top}=$ $\left(q^{\top}, p^{\top}\right)$ and $z^{\top}=\left(x^{\top}, y^{\top}\right)$, then we have to check that $\{\zeta, \zeta\}=J_{4},\{\zeta, I\}=0,\{\zeta, \theta\}=0$ and $\{\theta, I\}=1$. If $f(\theta, x, \xi, y)$ and $g(\theta, x, \xi, y)$ are functions taking vectorial values, we define the matrix of Poisson brackets $\{f, g\}$ as

$$
\{f, g\}=\frac{\partial f}{\partial z} J_{4}\left(\frac{\partial g}{\partial z}\right)^{\top}+\frac{\partial f}{\partial \theta}\left(\frac{\partial g}{\partial \xi}\right)^{\top}-\frac{\partial f}{\partial \xi}\left(\frac{\partial g}{\partial \theta}\right)^{\top}
$$

These equalities are easily verified using the symplectic character of $B(\theta)$ and the explicit expressions $\frac{\partial \zeta}{\partial z}=B(\theta)^{-1}, \frac{\partial \zeta}{\partial \theta}=-B(\theta)^{-1} B^{\prime}(\theta) B(\theta)^{-1} z, \frac{\partial \zeta}{\partial \xi}=0, \frac{\partial I}{\partial z}=-z^{\top} J_{4} B^{\prime}(\theta) B(\theta)^{-1}$ (this symplectic character implies that $J B^{\prime}(\theta) B(\theta)^{-1}$ is also a symmetric matrix) and $\frac{\partial I}{\partial \xi}=1$, that are computed from (25).

\subsubsection{The reduced Hamiltonian}

Inserting (25) into the Hamiltonian (21), we obtain (we keep the name $H$ ):

$$
H(\theta, x, \xi, y)=h_{0}+\omega_{0} \xi+\frac{\omega_{1}}{2}\left(x_{1}^{2}+y_{1}^{2}\right)+\frac{\omega_{2}}{2}\left(x_{2}^{2}+y_{2}^{2}\right)+\sum_{j \geq 3} H_{j}(\theta, x, \xi, y)
$$

being $\omega_{j}=\alpha_{j} / T, j=1,2$. Note that after this change the quadratic part of $(26)$ is reduced to constant coefficients.

\subsection{Complexification of the Hamiltonian}

With the Hamiltonian (26) we have a good system of coordinates to start computing the normal form. Nevertheless, to solve in a simpler form the different homological equations that will appear, it is much better to put the quadratic part of the Hamiltonian in diagonal form. For this purpose, we introduce new (complex) variables

$$
x_{j}=\left(Q_{j}+i P_{j}\right) / \sqrt{2}, \quad y_{j}=\left(i Q_{j}+P_{j}\right) / \sqrt{2}, \quad j=1,2 .
$$

We note that these relations define a canonical change that transforms the Hamiltonian (26) into

$$
H(\theta, Q, \xi, P)=h_{0}+\omega_{0} \xi+i \omega_{1} Q_{1} P_{1}+i \omega_{2} Q_{2} P_{2}+\sum_{j \geq 3} H_{j}(\theta, Q, \xi, P)
$$

keeping again the name for $H$. This is the expression of the Hamiltonian that we will use to start the nonlinear part of the normal form. Note that the image of the real domain for $x_{j}$ and $y_{j}$ in the complex variables $Q_{j}$ and $P_{j}$, is given by the relation $\bar{P}_{j}=i Q_{j}$. Hence, if 
this property is preserved during the normal form computation, we will be able to return to a real analytic Hamiltonian by means of the inverse of the change (27),

$$
Q_{j}=\left(x_{j}-i y_{j}\right) / \sqrt{2}, \quad P_{j}=\left(-i x_{j}+y_{j}\right) / \sqrt{2}, \quad j=1,2,
$$

In this context, the variables $(Q, P)$ in $(29)$ denote the current variables obtained after the different normal form transformations, and then, $(x, y)$ are the transformed variables of the initial real ones.

Returning to the complexified Hamiltonian (28), we change the previous notation to a more suitable one to describe the normal form. We write (28) as

$$
H^{(0)}(\theta, q, I, p)=h_{0}+\omega_{0} I+i \omega_{1} q_{1} p_{1}+i \omega_{2} q_{2} p_{2}+\sum_{j \geq 3} H_{j}^{(0)}(\theta, q, I, p),
$$

We note that this Hamiltonian has the following symmetry coming from the complexification (see [25]): if we expand $H^{(0)}$ as $f$ in (4) and (5), we have

$$
\bar{h}_{j, k, l, m, s}^{(0)} i^{|l|_{1}+|m|_{1}}=h_{j, k, m, l,-s}^{(0)} .
$$

\subsection{Computing the normal form}

The objective of this section is to put the Hamiltonian (30) in normal form up to finite order, by using a canonical change of variables $(2 \pi$-periodic in $\theta)$. We will construct this change as a composition of time one flows associated to suitable Hamiltonians (generating functions) $G_{j}$. They are selected to remove, in recursive form, the non-resonant terms of degree $j$. So, we will compute $G_{3}, G_{4}, \ldots, G_{n}$, such that:

$$
\begin{aligned}
H^{(n-2)} \equiv & H^{(0)} \circ \Phi_{t=1}^{G_{3}} \circ \cdots \circ \Phi_{t=1}^{G_{n}}(\theta, q, I, p)=h_{0}+\omega_{0} I+i \omega_{1} q_{1} p_{1}+i \omega_{2} q_{2} p_{2}+ \\
& +N^{(n)}\left(I, i q_{1} p_{1}, i q_{2} p_{2}\right)+H_{n+1}^{(n-2)}(\theta, q, I, p)+H_{n+2}^{(n-2)}(\theta, q, I, p)+\cdots
\end{aligned}
$$

where $\Phi_{t}^{G}$ means the flow time $t$ associated to the Hamiltonian system $G$. Here $N^{(n)}$ is in normal form up to order $n$, that is, only contains exactly resonant terms (unavoidable resonances) of degree not bigger than $n$.

Before describing how to obtain this normal form, let us mention two important properties of the Poisson bracket.

1. If $f$ and $g$ only contain monomials of degree $r$ and $s$ respectively, then $\{f, g\}$ only contains monomials of degree $r+s-2$.

2. If the expansions of $f$ and $g$ verify the symmetry (31), $\{f, g\}$ too.

The last property will guarantee that, after the normal form process, the change (29) will transform the final Hamiltonian into a real analytic one.

\subsubsection{A general step}

Let us describe one step of this normal form process. For this purpose, we take the Hamiltonian (32), and we compute $G_{n+1}$ by imposing that the expression

$$
\left\{\omega_{0} I+i \omega_{1} q_{1} p_{1}+i \omega_{2} q_{2} p_{2}, G_{n+1}\right\}+H_{n+1}^{(n-2)},
$$


only contains exactly resonant terms. Then, doing for $G_{n+1}$ and $H_{n+1}^{(n-2)}$ the same expansions as in (4) and (5), we formally obtain

$$
g_{n+1, k, l, m, s}=\frac{h_{n+1, k, l, m, s}^{(n-2)}}{i(l-m)^{\top} \omega+i s \omega_{0}},
$$

being $\omega^{\top}=\left(\omega_{1}, \omega_{2}\right)$, provided that the denominators do not vanish. The well-defined character of $G_{n+1}$ is ensured asking the denominators that do not correspond to trivial resonances to satisfy a suitable Diophantine condition (see [14]), to guarantee the convergence of the generating function $G_{n+1}$. The exactly resonant monomials correspond to $m=l$ and $s=0$, and they can not be removed (they are the only ones present in the normal form). Moreover, we remark that the coefficients $g_{n+1, k, l, m, s}$ satisfy the symmetry (31). Finally, as the selection of the coefficients $g_{n+1, k, l, l, 0}$ is free, in a practical implementation we will take $g_{n+1, k, l, l, 0}=0$, to have minimal norm for $G_{n+1}$ and to keep the symmetry (31). Then, applying the transformation $\Phi_{t=1}^{G_{n+1}}$ on the Hamiltonian $H^{(n-2)}$, we obtain

$$
H^{(n-1)} \equiv H^{(n-2)} \circ \Phi_{t=1}^{G_{n+1}}=H^{(n-2)}+\left\{H^{(n-2)}, G_{n+1}\right\}+\frac{1}{2 !}\left\{\left\{H^{(n-2)}, G_{n+1}\right\}, G_{n+1}\right\}+\cdots,
$$

that is in normal form up to order $n+1$, and it also satisfies the symmetry (31). Then, given a fixed $N \geq 3$, if we finish the process after $N-3$ normal form steps, we obtain a Hamiltonian of the form:

$$
\mathcal{H}(\theta, q, I, p) \equiv H^{(N-3)}=\mathcal{N}\left(I, i q_{1} p_{1}, i q_{2} p_{2}\right)+\mathcal{R}(\theta, q, I, p)
$$

where $\mathcal{N}$ is in normal form up to order $N-1$, and $\mathcal{R}$ is a remainder of order $N$.

\subsubsection{Changes of variables}

In practical computations one is usually interested in obtaining an explicit expression of the transformation that brings Hamiltonian (30) into its normal form (33),

$$
\Psi^{(N-3)} \equiv \Phi_{t=1}^{G_{3}} \circ \cdots \circ \Phi_{t=1}^{G_{N-1}}(\theta, q, I, p),
$$

as well as its inverse transformation

$$
\left(\Psi^{(N-3)}\right)^{-1}=\Phi_{t=-1}^{G_{N-1}} \circ \cdots \circ \Phi_{t=-1}^{G_{3}}=\Phi_{t=1}^{-G_{N-1}} \circ \cdots \circ \Phi_{t=1}^{-G_{3}} .
$$

To this end, we consider a generic (analytic) generating function $G(\theta, q, I, p), 2 \pi$-periodic on $\theta$, with a Taylor expansion starting with monomials of degree 3 , and a function $F(\theta, q, I, p)$ taking one of the following forms:

$$
\begin{aligned}
& F=q_{1}+f(\theta, q, I, p), \\
& F=I+f(\theta, q, I, p), \\
& F=\theta+f(\theta, q, I, p),
\end{aligned}
$$

being $f$ an analytic function, $2 \pi$-periodic on $\theta$, and with a Taylor expansion starting with terms of degree 2 in (35), 3 in (36) and 1 in (37), respectively. Now we will describe how to compute $F \circ \Phi_{t=1}^{G}$ using the Lie series method and, hence, putting initially $f \equiv 0$ in (35), 
(36) and (37), the different components of $\Psi^{(N-3)}$ are obtained by computing recursively $F \circ \Phi_{t=1}^{G_{3}} \circ \cdots \circ \Phi_{t=1}^{G_{N-1}}$. Note that to compute the transformation for $q_{2}, p_{1}$ or $p_{2}$, one only has to replace in (35) $q_{1}$ by one of these variables.

Let us start, for instance, with expression (35). We put $F_{1} \equiv F \circ \Phi_{t=1}^{G}=q_{1}+f_{1}$ and then, one has

$$
f_{1}=f+\{F, G\}+\frac{1}{2 !}\{\{F, G\}, G\}+\cdots .
$$

If $G$ begins with terms of degree 3 and $f$ of degree 2 we have, from the homogeneity of $\{.,$.$\} with respect to the adapted definition of degree, that f_{1}$ also begins with degree 2 . An analogous remark holds if we compute $f_{1}$ for $(36)$. In this case, we have that $f_{1}$ begins with terms of order at least 3. Finally, the transformation of (37) is a little bit different. To do it, we define $f^{*}$ as

$$
f^{*}=\{f, G\}+\frac{\partial G}{\partial I}
$$

Note that it begins with degree at least 1 . Then

$$
f_{1}=f+f^{*}+\frac{1}{2 !}\left\{f^{*}, G\right\}+\frac{1}{3 !}\left\{\left\{f^{*}, G\right\}, G\right\}+\cdots,
$$

that it is still of order 1 . Similar ideas are used to compute $\left(\Psi^{(N-3)}\right)^{-1}$.

\subsection{The normal form}

Let us consider the normal form $\mathcal{N}$ of (33). In order to go back to real coordinates we apply the change

$$
q_{j}=\left(x_{j}-i y_{j}\right) / \sqrt{2}, \quad p_{j}=\left(-i x_{j}+y_{j}\right) / \sqrt{2}, j=1,2,
$$

as it has been mentioned in (29). Then, $\mathcal{N}$ takes the form $\mathcal{N}\left(I_{0}, I_{1}, I_{2}\right)$, where the actions

$$
I_{0}=I, \quad I_{j}=i q_{j} p_{j}=\frac{x_{j}^{2}+y_{j}^{2}}{2}, j=1,2
$$

are first integrals for the Hamiltonian equations of $\mathcal{N}$. Moreover, as the changes of variables used for the normal form computation preserve the symmetry (31), we have that $\mathcal{N}\left(I_{0}, I_{1}, I_{2}\right)$ is a real analytic function (in fact, $\mathcal{N}$ is a polynomial of degree $[N / 2]$ ). Then, if we put $\omega_{j}\left(I_{0}, I_{1}, I_{2}\right) \equiv \frac{\partial \mathcal{N}}{\partial I_{j}}, j=0,1,2$, the solutions of the Hamiltonian equations of $\mathcal{N}$ are explicitly given by

$$
\begin{aligned}
\theta(t) & =\omega_{0}^{0} t+\theta^{0}, & x_{j}(t) & =\sqrt{2 I_{j}^{0}} \sin \left(\omega_{j}^{0} t+\theta_{j}^{0}\right), \\
I_{0}(t) & =I_{0}^{0}, & y_{j}(t) & =\sqrt{2 I_{j}^{0}} \cos \left(\omega_{j}^{0} t+\theta_{j}^{0}\right),
\end{aligned}
$$

for $j=1,2$, where $\omega_{j}^{0} \equiv \omega_{j}\left(I_{0}^{0}, I_{1}^{0}, I_{2}^{0}\right)$.

\subsubsection{Invariant tori}

From the normal form obtained in Section 2.6, it is easy to produce approximations to periodic orbits and invariant tori of dimensions 2 and 3, as well as approximations of their intrinsic and normal frequencies. They are obtained neglecting the remainder of the 
Hamiltonian and selecting values for the actions in a sufficiently small neighbourhood of the periodic orbit.

More concretely, if we put $I_{j}^{0}=0, j=1,2$, in (40), we have parameterized by $I_{0}^{0}$ a 1-parameter family of periodic orbits (that contains the initial one for $I_{0}^{0}=0$ ). By putting $I_{1}^{0}=0$, we obtain a 2-parameter family of 2-dimensional tori, parameterized by $I_{0}^{0}$ and $I_{2}^{0}$. We have a symmetric situation swapping $I_{1}^{0}$ by $I_{2}^{0}$. If we use the three parameters simultaneously, $I_{j}^{0} j=0,1,2$, we describe in (40) a 3 -parameter family of 3 -dimensional tori.

If we send these tori along the different normal form transformations (see Section 2.5.2), complexified coordinates (Section 2.4), the Floquet transformation (Section 2.3) and the adapted coordinates (Section 2.1), we obtain approximations of periodic orbits and invariant tori for the initial system (1).

Similar ideas can be used, for example, in the case of periodic orbits with some hyperbolic directions to compute approximations of hyperbolic tori and the corresponding stable and unstable manifols. The main difference withthe case we are actually dealing with, appears in the Floquet transformation, where it is necessary to take into account the hyperbolic eigenvalues. We recall (see Section 2.3) that in some cases these hyperbolic directions can be an obstruction for the determination of a real Floquet transformation.

\subsubsection{Effective stability}

Normal form computations are also useful to derive bounds on the diffusion speed near some invariant objects. It is well known that, in Hamiltonian systems with more than 2 degrees of freedom, linear stability does not imply stability (see, for instance, [2] for a first description of a model for this unstability), but accurate bounds on the diffusion velocity show that it must be very slow (see references in the Introduction). This leads to the introduction of the concept of effective stability (see [8]): An object is called $\sigma$-stable $(\sigma>1)$ up to time $T$, if there exists $\tau>0$ such that any solution starting at distance $\tau$ of the invariant object remains at distance not great than $\sigma \tau$ up to, at least, time $T$.

This kind of stability can be obtained from the normal form we have constructed here. One only needs to derive good estimates for the remainder of the normal form (this is the part of the Hamiltonian that can produce the diffusion) to derive the desired estimates. Next sections are devoted to describe how these estimates can be obtained.

\subsection{Bounds on the domain of convergence of the normal form}

Now, we will estimate the size of the region of effective stability around the periodic orbit. To determine this region we use the following criteria: we identify the region of slow diffusion with the domain around the orbit where we can prove that the normal form up to order "big enough" (in a practical implementation, this is usually the biggest order one can reach within the computer limitations) is convergent with a sufficiently small remainder.

To implement the previous approach, we will give a method to bound the domain where the changes that introduce the normal form coordinates (see Section 2.5.2) are convergent. For this purpose we consider, as in Section 2.5.2, a generic (analytic) generating function $G(\theta, q, I, p)$. Then, if one puts $\Phi_{t}^{G}(\theta(0), q(0), I(0), p(0))=(\theta(t), q(t), I(t), p(t))$ for the 
time $t$ flow associated to the Hamiltonian equations of $G$, one can write

$$
\begin{aligned}
\theta(t) & =\theta(0)+\int_{0}^{t} \frac{\partial G}{\partial I}(\theta(s), q(s), I(s), p(s)) d s \\
I(t) & =I(0)-\int_{0}^{t} \frac{\partial G}{\partial \theta}(\theta(s), q(s), I(s), p(s)) d s \\
\left(\begin{array}{l}
q(t) \\
p(t)
\end{array}\right) & =\left(\begin{array}{c}
q(0) \\
p(0)
\end{array}\right)+J_{4} \int_{0}^{t}\left(\frac{\partial G}{\partial(q, p)}\right)^{\top}(\theta(s), q(s), I(s), p(s)) d s .
\end{aligned}
$$

To estimate the region where the transformation $\Phi_{t=1}^{G}$ is defined, we use the norm $\|\cdot\|_{\rho, R}$ introduced in (9). Hence, it is not difficult to deduce from the integral expressions (41), (42) and (43), that if one puts

$$
\rho_{0}=\left\|\frac{\partial G}{\partial I}\right\|_{\rho^{(0)}, R^{(0)}}, \quad \delta_{0}=\left\|\frac{\partial G}{\partial \theta}\right\|_{\rho^{(0)}, R^{(0)}}, \delta_{j}=\left\|\frac{\partial G}{\partial p_{j}}\right\|_{\rho^{(0)}, R^{(0)}}, \delta_{j+2}=\left\|\frac{\partial G}{\partial q_{j}}\right\|_{\rho^{(0)}, R^{(0)}},
$$

for $j=1,2$, and one assumes $\rho_{0}<\rho^{(0)}$ and $\delta_{j}<R_{j}^{(0)}, j=0, \ldots, 4$, then $\Phi_{t=1}^{G}$ is well defined from $\mathcal{D}\left(\rho^{(0)}-\rho_{0}, R^{(0)}-\delta\right)$ to $\mathcal{D}\left(\rho^{(0)}, R^{(0)}\right)$, where $\delta=\left(\delta_{0}, \ldots, \delta_{4}\right)$ (see $(7)$ for the definition of $\mathcal{D}(.,)$.$) . The proof can be found in [5]$.

\subsection{Bounds on the diffusion speed}

To apply the ideas described along Section 2 to a practical example, we modify the normal form method introduced in Section 2.5, to adapt it to the standard implementation of normal forms in a computer, where it is usual to work with (truncated) power series stored using the standard definition of degree of a monomial, instead of the adapted one made in $(6)$.

To avoid confusions, in what follows the word "degree" will refer to the adapted degree defined in (6), while "standard degree" will refer to the usual degree for monomials. Hence, instead of using generating functions $G_{j}$ that are homogeneous polynomials of degre $j$, we will use generating functions that are homogeneous polynomials of standard degree $j$. With this formulation the remainder $\mathcal{R}$ of (33) begins with terms of standard degree $N$. As the normal form is independent of the process used to compute it, the only difficult that we find if we do not use the adapted definition of degree is of technical character: if we work with the standard degree, and we perform the Poisson bracket of two monomial of degree $r$ and $s$, we loose the homogeneity of the Poisson bracket, and the result contains terms of degree $r+s-1$ and $r+s-2$. Note that, although this is not very nice for theoretical purposes, it is not a problem for a computational scheme. ${ }^{1}$

We use the criteria given in Section 2.7 to compute the effective stability region: we are interested in obtaining a domain where the canonical transformation $\Psi^{(N-3)}$ of $(34)$ is convergent. To this end, we define (for technical reasons) $G \equiv G_{3}+G_{4}+\cdots+G_{N-3}$. Then, given an initial domain $\mathcal{D}\left(\rho^{(0)}, R^{(0)}\right)$ (small enough) where we expect $\Psi^{(N-3)}$ to be convergent, we compute $\rho_{0}$ and the vector $\delta$ given by (44), using the definition of $G$ previously done, but replacing the norm $\|\cdot\|_{\rho^{(0)}, R^{(0)}}$ by $|\cdot|_{\rho^{(0)}, R^{(0)}}$. It is not difficult to check (using the bounds given in Section 2.7 on any transformation $G_{j}, j=N-3, N-2, \ldots, 3$ )

\footnotetext{
${ }^{1}$ In fact, it is also possible to perform all the computations using the adapted degree, with a similar amount of work.
} 
that $\Psi^{(N-3)}$ is convergent from $\mathcal{D}(\rho, R)$ to $\mathcal{D}\left(\rho^{(0)}, R^{(0)}\right)$, where $\rho=\rho^{(0)}-\rho_{0}$ and $R=$ $R^{(0)}-\delta$, provided that the initial domain is small enough such that $\rho>0$ and $R_{j}>0$, $j=0, \ldots, 4$.

To bound the diffusion speed on $\mathcal{D}(\rho, R)$, we assume that we know $M \geq 0$ such that $\left\|H^{(0)}\right\|_{\rho^{(0)}, R^{(0)}} \leq M$ (that is, a bound for the norm of the Hamiltonian used to begin the normal form computations). So, for the Hamiltonian $\mathcal{H}$ of (33) we also have that $\|\mathcal{H}\|_{\rho, R} \leq M$.

Then, what we are going to do is to take arbitrary initial data in $\mathcal{D}(0, R)$, with the restriction that these points correspond to a representation of real points expressed in the complexified variables introduced in (27), and to estimate the time that the solution of the Hamiltonian equations corresponding to $\mathcal{H}$ needs to increase the distance to the initial periodic orbit in a given amount, at least, until this solution leaves $\mathcal{D}(0, R)$. In fact, we are going to produce bounds for this time as a function of the initial and final distance to the periodic orbit.

For this purpose, we consider the canonical equations for $(I, q, p)$ related to $\mathcal{H}$. From (33), and using the notation for $I_{0}, I_{1}$ and $I_{2}$ introduced in (39), we have:

$$
\begin{aligned}
\dot{q}_{j} & =\frac{\partial \mathcal{N}}{\partial I_{j}} i q_{j}+\frac{\partial \mathcal{R}}{\partial p_{j}} \\
\dot{p}_{j} & =-\frac{\partial \mathcal{N}}{\partial I_{j}} i p_{j}-\frac{\partial \mathcal{R}}{\partial q_{j}}
\end{aligned}
$$

for $j=1,2$, and

$$
\dot{I}_{0}=-\frac{\partial \mathcal{N}}{\partial \theta}-\frac{\partial \mathcal{R}}{\partial \theta}=-\frac{\partial \mathcal{R}}{\partial \theta}
$$

where we recall that $\mathcal{N} \equiv \mathcal{N}\left(I_{0}, i q_{1} p_{1}, i q_{2} p_{2}\right)$. We do not consider the "diffusion" in the $\theta$-direction, as it does not increase the distance from the initial periodic orbit. From (45), (46) and (47), one obtains

$$
\begin{aligned}
& \dot{I}_{0}=-\frac{\partial \mathcal{R}}{\partial \theta} \equiv \hat{I}_{0}(\theta, q, I, p) \\
& \dot{I}_{j}=i\left(\frac{\partial \mathcal{R}}{\partial p_{j}} p_{j}-\frac{\partial \mathcal{R}}{\partial q_{j}} q_{j}\right) \equiv \hat{I}_{j}(\theta, q, I, p), \quad j=1,2 .
\end{aligned}
$$

We remark that, to bound (48) and (49), we do not have explicit bounds for the remainder $\mathcal{R}$, but we recall that it begins with terms of standard degree $N$. As we know a bound $M$ for the transformed Hamiltonian $\mathcal{H}$ in the domain $\mathcal{D}(\rho, R)$, we can compute (using Cauchy estimates) a bound for the remainder of its Taylor series up to standard degree $N-1$, that is, a bound for $\mathcal{R}$.

We use this idea to bound the right-hand sides of (48) and (49). To do that, we define $R_{0}^{*}=R_{0}, R_{1}^{*}=\min \left\{R_{1}, R_{3}\right\}$ and $R_{2}^{*}=\min \left\{R_{2}, R_{4}\right\}$, and we consider real points $(\theta, x, I, y)$, using the variables $\left(x_{j}, y_{j}\right)$ introduced in $(38)$, such that $\left|I_{0}\right|<R_{0}^{*}$ and $I_{j}<$ $\left(R_{j}^{*}\right)^{2}$. From definition (38), we note that the set of real points $\left(x_{j}, y_{j}\right)$ such that $I_{j} \leq I_{j}^{(0)}$, is contained in the complex set $\left\{\left|q_{j}\right|,\left|p_{j}\right| \leq \sqrt{I_{j}^{(0)}}\right\}, j=1,2$. Then, applying the Cauchy estimates given in (12) and (13), to the coefficients of the Taylor expansion of $\hat{I}_{0}$ and $\hat{I}_{j}$, $j=1,2$, we deduce the bounds

$$
\left|\hat{I}_{0}\right| \leq \frac{1}{\rho} \sum_{m \in \mathbb{N}^{5},|m|_{1} \geq N} \frac{M\left|I_{0}\right|^{m_{0}}\left(\sqrt{I_{1}}\right)^{m_{1}+m_{3}}\left(\sqrt{I_{2}}\right)^{m_{2}+m_{4}}}{\left(R_{0}^{*}\right)^{m_{0}}\left(R_{1}^{*}\right)^{m_{1}+m_{3}}\left(R_{2}^{*}\right)^{m_{2}+m_{4}}}
$$




$$
\begin{aligned}
\left|\hat{I}_{j}\right| & \leq \sum_{m \in \mathbb{N}^{5},|m|_{1} \geq N}\left(m_{j}+m_{j+2}\right) \frac{M\left|I_{0}\right|^{m_{0}}\left(\sqrt{I_{1}}\right)^{m_{1}+m_{3}}\left(\sqrt{I_{2}}\right)^{m_{2}+m_{4}}}{\left(R_{0}^{*}\right)^{m_{0}}\left(R_{1}^{*}\right)^{m_{1}+m_{3}}\left(R_{2}^{*}\right)^{m_{2}+m_{4}}}= \\
& =\sum_{m \in \mathbb{N}^{5},|m|_{1} \geq N} 2 m_{1} \frac{M\left|I_{0}\right|^{m_{0}}\left(\sqrt{I_{1}}\right)^{m_{1}+m_{3}}\left(\sqrt{I_{2}}\right)^{m_{2}+m_{4}}}{\left(R_{0}^{*}\right)^{m_{0}}\left(R_{1}^{*}\right)^{m_{1}+m_{3}}\left(R_{2}^{*}\right)^{m_{2}+m_{4}}}, j=1,2,
\end{aligned}
$$

being $m=\left(m_{0}, m_{1}, \ldots, m_{5}\right)$ in these sums. To bound (50) and (51) uniformily, we define

$$
\delta=\delta\left(I_{0}, I_{1}, I_{2}\right) \equiv \max \left\{\frac{\left|I_{0}\right|}{R_{0}^{*}}, \frac{\sqrt{I_{1}}}{R_{1}^{*}}, \frac{\sqrt{I_{2}}}{R_{2}^{*}}\right\} .
$$

We note that $\delta$ is an estimate for the distance to the initial periodic orbit. When $I_{0}, I_{1}$ and $I_{2}$ move as a function of $t$, we can also consider $\delta \equiv \delta(t)$. Then, from the definition of $\delta$, we can bound, for $\delta \leq 1$,

$$
\left|\hat{I}_{0}\right| \leq \frac{M}{\rho} \sum_{m \in \mathbb{N}^{5},|m|_{1} \geq N} \delta^{|m|_{1}} \leq \frac{M}{\rho}\left(\begin{array}{c}
4+N \\
4
\end{array}\right) \frac{\delta^{N}}{(1-\delta)^{N+5}},
$$

and

$$
\left|\hat{I}_{1}\right| \leq 2 M \sum_{m \in \mathbb{N}^{5},|m|_{1} \geq N} m_{1} \delta^{|m|_{1}} \leq 2 M\left(\begin{array}{c}
4+N \\
5
\end{array}\right) \frac{\delta^{N}}{(1-\delta)^{N+5}}, \quad j=1,2 .
$$

To bound the sums (53) and (54), we have used Lemma 2, that is given at the end of this section. Then, if we assume $\left|I_{0}\right|, I_{1}$ and $I_{2}$ to be increasing functions of $t$ (this is the worst case to bound the diffusion), we have:

$$
\frac{d}{d t}\left|\frac{I_{0}^{2}}{\left(R_{0}^{*}\right)^{2}}\right| \leq \frac{2\left|I_{0}\right|\left|\dot{I}_{0}\right|}{\left(R_{0}^{*}\right)^{2}} \leq \frac{2 \delta}{R_{0}^{*}} \frac{M}{\rho}\left(\begin{array}{c}
4+N \\
4
\end{array}\right) \frac{\delta^{N}}{(1-\delta)^{N+5}} \equiv A \frac{\delta^{N+1}}{(1-\delta)^{N+5}},
$$

where

$$
A=\frac{2}{R_{0}^{*}} \frac{M}{\rho}\left(\begin{array}{c}
4+N \\
4
\end{array}\right)
$$

and

$$
\frac{d}{d t}\left|\frac{I_{j}}{\left(R_{j}^{*}\right)^{2}}\right| \leq \frac{2 M}{\left(R_{j}^{*}\right)^{2}}\left(\begin{array}{c}
4+N \\
5
\end{array}\right) \frac{\delta^{N}}{(1-\delta)^{N+5}} \equiv B_{j} \frac{\delta^{N}}{(1-\delta)^{N+5}}
$$

with

$$
B_{j}=\frac{2 M}{\left(R_{j}^{*}\right)^{2}}\left(\begin{array}{c}
4+N \\
5
\end{array}\right)
$$

Putting $B=\max \left\{B_{1}, B_{2}\right\}$, we deduce the following bound for the speed of $\delta$ :

$$
\frac{d}{d t}\left(\delta^{2}\right) \leq \max \{A \delta, B\} \frac{\delta^{N}}{(1-\delta)^{N+5}},
$$

for $0<\delta<1$. Then, the problem of bounding the stability time can be solved in the following form: we take initial data corresponding to real coordinate points $(\theta, x, I, y)$, such that the corresponding $I_{j}$ are bounded by $I_{j}^{(0)}, j=0,1,2$. Let us assume that $\delta_{0} \equiv \delta\left(I_{0}^{(0)}, I_{1}^{(0)}, I_{2}^{(0)}\right)<1$. Given $\delta_{1}, \delta_{0}<\delta_{1} \leq 1$, we want a lower bound for the time 
$T\left(\delta_{0}, \delta_{1}\right)$ needed for the value of $\delta$ along the trajectory to go from $\delta_{0}$ to $\delta_{1}$. For this purpose, we take as initial condition $\delta(0)=\delta_{0}$, and we integrate

$$
\dot{\delta}=\frac{A}{2} \frac{\delta^{N}}{(1-\delta)^{N+5}}
$$

or

$$
\dot{\delta}=\frac{B}{2} \frac{\delta^{N-1}}{(1-\delta)^{N+5}},
$$

depending on the current value of $\delta$. Let us explain the method. We define $\delta^{*}=B / A$, and hence, if $\delta_{0} \geq \delta^{*}$, we use equation (55) for $\dot{\delta}$. If $\delta_{0}<\delta^{*}$, we use (56) for $0<t \leq T^{*}$, where $T^{*}$ is the value of $t$ for which the bound of $\delta(t)$ obtained integrating (56) reachs $\delta^{*}$. Then, for $t>T^{*}$, we use the bound (55) (of course, it can happen that $\delta^{*}>1$ ). For (55), the necessary time $\Delta t$ to move from $\delta_{i}$ to $\delta_{f}$ is given by:

$$
\begin{aligned}
\Delta t= & \frac{2}{A} \int_{\delta_{i}}^{\delta_{f}} \frac{(1-\delta)^{N+5}}{\delta^{N}} d \delta=\left.\frac{2}{A} \sum_{\substack{j=0 \\
j \neq N-1}}^{N+5}\left(\begin{array}{c}
N+5 \\
j
\end{array}\right)(-1)^{j} \frac{\delta^{j-N+1}}{j-N+1}\right|_{\delta_{i}} ^{\delta_{f}}+ \\
& +\left.\frac{2}{A}\left(\begin{array}{c}
N+5 \\
N-1
\end{array}\right)(-1)^{N-1} \log (\delta)\right|_{\delta_{i}} ^{\delta_{f}},
\end{aligned}
$$

and using (56), this time is

$$
\begin{aligned}
\Delta t & =\frac{2}{B} \int_{\delta_{i}}^{\delta_{f}} \frac{(1-\delta)^{N+5}}{\delta^{N-1}} d \delta=\left.\frac{2}{B} \sum_{\substack{j=0 \\
j \neq N-2}}^{N+5}\left(\begin{array}{c}
N+5 \\
j
\end{array}\right)(-1)^{j} \frac{\delta^{j-N+2}}{j-N+2}\right|_{\delta_{i}} ^{\delta_{f}}+ \\
& +\left.\frac{2}{B}\left(\begin{array}{c}
N+5 \\
N-2
\end{array}\right)(-1)^{N-2} \log (\delta)\right|_{\delta_{i}} ^{\delta_{f}} .
\end{aligned}
$$

To end this section, we formulate and prove the result used to sum the bounds for the diffusion speed in (53) and (54). For this purpose, we define $c_{n, l}=\#\left\{m \in \mathbb{N}^{n}:|m|_{1}=l\right\}$, that is, the number of monomials in $n$ variables of degree $l$. This number is given by

$$
c_{n, l}=\left(\begin{array}{c}
n+l-1 \\
n-1
\end{array}\right)
$$

Lemma 2 For any $0 \leq R<1$, we have the following bounds:

$(i)$

$$
\sum_{m \in \mathbb{N}^{n},|m|_{1} \geq N} R^{|m|_{1}}=\sum_{l \geq N} c_{n, l} R^{l} \leq\left(\begin{array}{c}
n+N-1 \\
n-1
\end{array}\right) \frac{R^{N}}{(1-R)^{N+n}}
$$

(ii)

$$
\sum_{m \in \mathbb{N}^{n},|m|_{1} \geq N} m_{1} R^{|m|_{1}}=\sum_{l \geq N} \sum_{j=0}^{l} j c_{n-1, l-j} R^{l} \leq\left(\begin{array}{c}
n+N-1 \\
n
\end{array}\right) \frac{R^{N}}{(1-R)^{N+n}}
$$

being, in both cases, $m=\left(m_{1}, \ldots, m_{n}\right)$. 
Proof: : We define $f(x)=(1-x)^{-n}=\sum_{l \geq 0} c_{n, l} x^{l}$. Then, part $(i)$ is obtained bounding the remainder of the Taylor expansion up to degree $N-1$ of $f$ around $x=0$, evaluated at $x=R$. To do that, we remark that differentiating $f j$ times, we have $f^{(j)}(x)=$ $n(n+1) \cdots(n+j-1)(1-x)^{-n-j}$ and, hence,

$$
\frac{f^{(N)}(x)}{N !} \leq\left(\begin{array}{c}
n+N-1 \\
N
\end{array}\right)(1-R)^{-N-n}
$$

for every $0<x \leq R$. To prove $(i i)$, we use that

$$
\sum_{j=0}^{l} j c_{n-1, l-j}=\left(\begin{array}{c}
n+l-1 \\
n
\end{array}\right)=c_{n+1, l-1},
$$

that is easily proved by induction. Finally, using the bound $(i)$, we obtain:

$$
\sum_{l \geq N} \sum_{j=0}^{l} j c_{n-1, l-j} R^{l}=\sum_{l \geq N} c_{n+1, l-1} R^{l}=R \sum_{l \geq N-1} c_{n+1, l} R^{l} \leq\left(\begin{array}{c}
n+N-1 \\
n
\end{array}\right) \frac{R^{N}}{(1-R)^{N+n}} .
$$

\section{Application to the spatial RTBP}

Here we present an application of the methods of Section 2 to a concrete example coming from the Restricted Three Body Problem (RTBP). As it will be explained in the following sections, we have taken an elliptic periodic orbit of the RTBP, we have computed (numerically) the normal form (up to order 16) and we have bounded the corresponding remainder. This normal form has been used to compute invariant tori (of dimensions 1, 2 and 3) near the periodic orbit, and the bounds on the remainder have been used to estimate the corresponding rate of diffusion.

\subsection{The Restricted Three Body Problem}

Let us consider two bodies (usually called primaries) revolving in circular orbits around their common centre of masses, under the action of Newton's law. With this, we can write the equations of motion of a third infinitessimal particle moving under the gravitational attraction of the primaries, but without affecting them. The study of the motion of this third particle is the so-called Restricted Three Body Problem (see [29]). To simplify the equations, the units of length, time and mass are chosen such that the sum of masses of the primaries, the distance between them and the gravitational constant are all equal to one. With these normalized units, the angular velocity of the primaries around their centre of masses is also equal to one. A usual system of reference (called synodical system) is the following: the origin is taken at the centre of mass of the two primaries, the $x$ axis is given by the line defined by the two primaries and oriented from the smaller primary to the biggest one, the $z$ axis has the direction of the angular momentum of the motion of the primaries and the $y$ axis is taken in order to have a positively oriented system of reference. If we suppose that the masses of the primaries are $\mu$ and $1-\mu$, with $0<\mu \leq 1 / 2$, we have that the primaries are located (in the synodic system) at the points $(\mu-1,0,0)$ 
and $(\mu, 0,0)$, respectively. In this reference, the Hamiltonian for the motion of the third particle is

$$
H\left(x, y, z, p_{x}, p_{y}, p_{z}\right)=\frac{1}{2}\left(p_{x}^{2}+p_{y}^{2}+p_{z}^{2}\right)+y p_{x}-x p_{y}-\frac{1-\mu}{r_{1}}-\frac{\mu}{r_{2}},
$$

being the momenta $p_{x}=\dot{x}-y, p_{y}=\dot{y}+x$ and $p_{z}=\dot{z}$, with $r_{1}^{2}=(x-\mu)^{2}+y^{2}+z^{2}$ and $r_{2}^{2}=(x-\mu+1)^{2}+y^{2}+z^{2}$. The parameter $\mu$ is usually called the mass parameter of the system. Note that the $(x, y)$ plane is invariant by the flow. The restriction of this Hamiltonian to this plane is the so-called planar RTBP, while (57) is usually called spatial RTBP. From now on, we will simply use RTBP to refer to the spatial problem.

This system has five equilibrium points: three of them are on the $x$ axis (usually called collinear points, or $L_{1}, L_{2}$ and $L_{3}$ ) and the other two are forming an equilateral triangle with the primaries (and are usually called triangular points, or $L_{4}$ and $L_{5}$ ). The points $L_{4}$ and $L_{5}$ are given in the phase space by $\left(-\frac{1}{2}+\mu,-\frac{\sqrt{3}}{2}, 0, \frac{\sqrt{3}}{2},-\frac{1}{2}+\mu, 0\right)$ and $\left(-\frac{1}{2}+\mu, \frac{\sqrt{3}}{2}, 0,-\frac{\sqrt{3}}{2},-\frac{1}{2}+\mu, 0\right)$.

\subsection{The vertical family of periodic orbits of $L_{5}$}

In what follows we will focus on the $L_{5}$ point, but the results are obviously true for $L_{4}$, due to the symmetries of the problem.

The linearized system around $L_{5}$ always has a vertical oscillation with frequency 1 . Then, the vertical family of periodic orbits is the Lyapunov family associated to this normal frequency (for a proof of the existence of these families, see [24]). To study the linear stability of these orbits, we recall that the eigenvalues of the linearized vectorfield at $L_{5}$ are given by $\pm i$ (the ones responsible of the vertical oscillation), and by

$$
\pm \sqrt{-\frac{1}{2} \pm \frac{1}{2} \sqrt{1-27 \mu(1-\mu)}}
$$

that are the ones of the planar RTBP. All of them are purely imaginary and different if $0<\mu<\mu_{R} \equiv \frac{1}{2}(1-\sqrt{23 / 27}) \approx 0.03852$ (this is the so-called Routh critical value). For $\mu=\mu_{R}$, the planar frequencies collide and this fact produces a bifurcation in the linear stability and $L_{5}$ becomes unstable for $\mu_{R} \leq \mu \leq 1 / 2$. Note that, if $\mu \neq \mu_{R}$, the linear character of the vertical family is the same as $L_{5}$, at least for small vertical amplitudes.

Now, we want to continue the vertical family of periodic orbits by increasing the amplitude. To do that, we identify the point $L_{5}$ with the periodic orbit of zero amplitude and period $2 \pi$. Hence, the monodromy matrix of this orbit is given by the exponential matrix of $2 \pi$ times the differential matrix of the RTBP vectorfield at $L_{5}$. For any periodic orbit of the vertical family, its monodromy matrix has, of course, a pair of eigenvalues 1 , plus other four eigenvalues that generalize the planar ones of $L_{5}$ to the vertical periodic orbits. As it has been mentioned in Section 2.3, the linear stability condition for these periodic orbits is that these four eigenvalues are all different and of modulus 1 . Returning to the case $\mu=\mu_{R}$, we have for the orbit of zero amplitude that these four eigenvalues collapse to two double eigenvalues in the complex unity circle. This resonance can be continued (numerically) with respect to $\mu$ and the amplitude of the orbit (in fact, it can be continued with respect to any regular parameter in the family). The curve corresponding to this resonance is displayed in Figure 1 . The variables plotted are $\mu$ and the vertical 

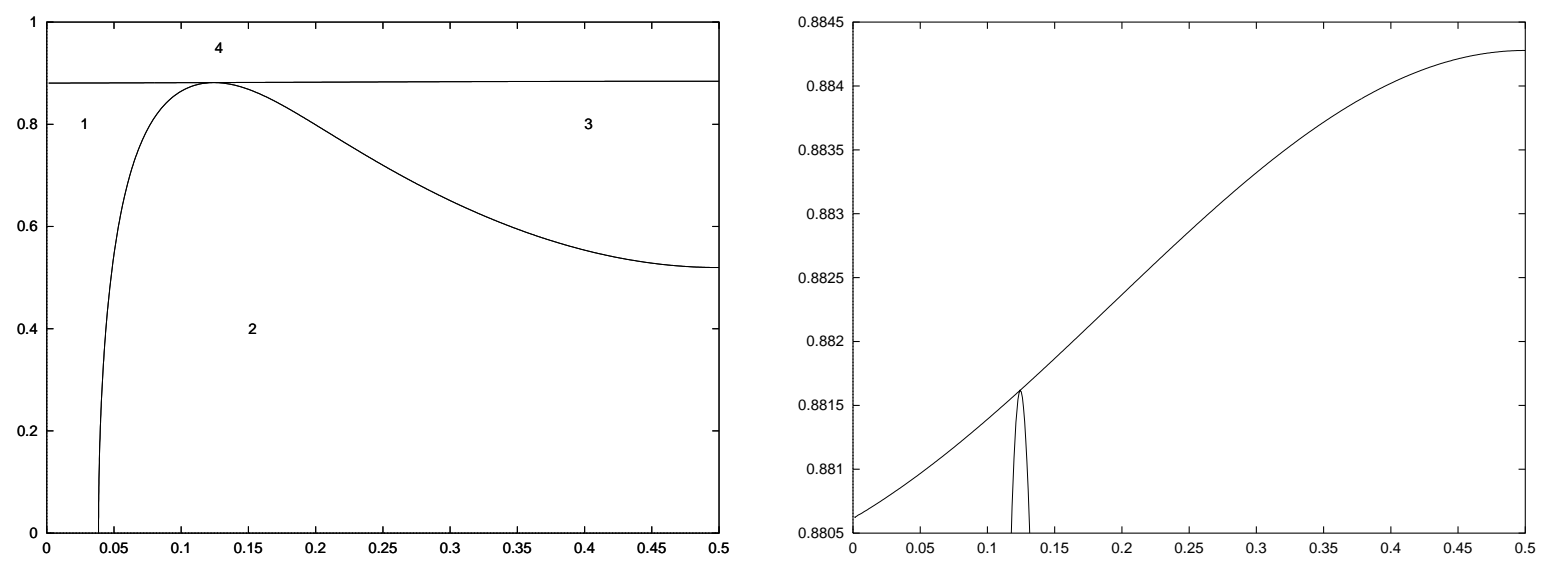

Figure 1: Left: Some curves of change of the linear character of the orbits of the vertical family of $L_{5}$. The variables in the plots are $\mu$ (horizontal axis)and the value of $\dot{z}$ (vertical axis) when $z=0$. Excluding the bifurcation curves, the normal eigenvalues of the periodic orbit are all different and can be described, according to the region, as follows: 1 . two couples of conjugate eigenvalues of modulus 1,2 . two conjugate eigenvalues ouside $\mathbb{S}^{1}$ and the corresponding inverse ones, 3. two couples of positive eigenvalues $\gamma, 1 / \gamma, 4$. two conjugate eigenvalues of modulus 1 and a couple of positive eigenvalues $\gamma, 1 / \gamma$, at least for moderate values of $\dot{z}$. Right: The upper curve with a more suitable scale for the $\dot{z}$ variable.

velocity $(\dot{z})$ of the orbit when it cuts the hyperplane $z=0$ in the positive direction. Note that, for values of $\mu$ slightly larger than $\mu_{R}, L_{5}$ is unstable but, if we "go up" in the vertical family, we find (linearly) stable orbits after crossing the above-mentioned bifurcation.

\subsubsection{The selected periodic orbit}

For the application of the methods exposed, we have selected a periodic orbit of the vertical family of $L_{5}$ for the mass parameter $\mu=0.04$ (that is bigger than $\mu_{R}$ ), and with $\dot{z}=0.24999973950378$. This is a linearly stable orbit (the corresponding pair $(\mu, \dot{z})$ belongs to region 1 in Figure 1, see Section 3.6 for more details), but its proximity to resonance will produce small domains of convergence for the normal form.

The reasons for selecting this orbit are the following. The vertical family of the RTBP has its own interest, since it is the skeleton that organizes the dynamics of some physically relevant problems (see [10], [28] and [27]), and the tools used here can be useful to deal with those problems. On the other hand, this example allows to show (numerically) the existence of regions of effective stability near $L_{4,5}$ for $\mu>\mu_{R}$. Finally, the example has not been artificially "cooked" to simplify computations so it is a good problem to test the effectivity of these techniques.

\subsection{Expansion of the Hamiltonian of the RTBP}

Let us denote by $\frac{2 \pi}{\omega_{0}}$ the period of the selected orbit. The next step is to perform the different changes of coordinates introduced in Section 2, and to compute the explicit expansion of the Hamiltonian expressed in these adapted coordinates (to obtain the Hamiltonian $H^{(0)}$ of (30), to start the computation of the normal form). 
For this purpose, we proceed in the following form. First, we write $\left(X, Y, Z, P_{X}, P_{Y}, P_{Z}\right)$ for the initial coordinates with origin at $L_{5}: X=x+\frac{1}{2}-\mu, Y=y-\frac{\sqrt{3}}{2}, Z=z$, $P_{X}=p_{x}+\frac{\sqrt{3}}{2}, P_{Y}=p_{y}+\frac{1}{2}-\mu$ and $P_{Z}=p_{z}$. Then, we assume that $(f(\theta), g(\theta))$ is a $2 \pi$-periodic parametrization of the periodic orbit, expressed in these coordinates. We note that, for orbits of this family with moderate amplitudes, the expression $\left(f_{3}^{\prime}\right)^{2}+\left(\frac{g_{3}^{\prime}}{\omega_{0}}\right)^{2}$ is close to a constant. More concretely, if we introduce the new coordinates $\zeta=\sqrt{\omega_{0}} Z$ and $P_{\zeta}=\frac{P_{Z}}{\sqrt{\omega_{0}}}$, the expressions for the new $f_{3}$ and $g_{3}$ take the form (2), with the function $\Delta$ of (3) close to constant. If we rewrite the Hamiltonian (57) in these new variables, but keeping for simplicity the notation $Z, P_{Z}$, then we can expand the Hamiltonian (57) as:

$$
\begin{aligned}
H & =\frac{1}{2}\left(P_{X}^{2}+P_{Y}^{2}+\omega_{0} P_{Z}^{2}\right)+Y P_{X}-X P_{Y}+\frac{X^{2}}{8}-\frac{5}{8} Y^{2}-a X Y+\frac{1}{2} \frac{Z^{2}}{\omega_{0}}- \\
& -\sum_{k \geq 3} r_{0}^{k} P_{k}\left(\frac{X-\sqrt{3} Y}{2 r_{0}}\right)(1-\mu)-\sum_{k \geq 3} r_{0}^{k} P_{k}\left(\frac{-X-\sqrt{3} Y}{2 r_{0}}\right) \mu,
\end{aligned}
$$

where we have skipped the constant term of this expansion, $\left(-3+\mu-\mu^{2}\right) / 2$, that corresponds to the energy level of $L_{5}$. Here, $a=-\frac{3 \sqrt{3}}{4}(1-2 \mu), r_{0}^{2}=X^{2}+Y^{2}+\frac{Z^{2}}{\omega_{0}}$ and $P_{k}$ denotes the Legendre polynomial of degree $k$. This expression comes from the expansion of the Hamiltonian of the RTBP around the point $L_{5}$. To expand (58) we can use the recurrence of the Legendre polynomial. This recurrence is given by $P_{0}(x)=1, P_{1}(x)=x$ and $P_{k+1}(x)=\frac{2 k+1}{k+1} x P_{k}(x)-\frac{k}{k+1} P_{k-1}(x)$, for $k \geq 1$, and hence, if we define:

$$
R_{k}^{(0)}=r_{0}^{k} P_{k}\left(\frac{X-\sqrt{3} Y}{2 r_{0}}\right), \quad R_{k}^{(1)}=r_{0}^{k} P_{k}\left(\frac{-X-\sqrt{3} Y}{2 r_{0}}\right),
$$

we have $R_{0}^{(0)}=1, R_{1}^{(0)}=\frac{X-\sqrt{3} Y}{2}$, and $R_{0}^{(1)}=1, R_{1}^{(1)}=\frac{-X-\sqrt{3} Y}{2}$. Thus,

$$
R_{k+1}^{(j)}=\frac{2 k+1}{k+1} R_{1}^{(j)} R_{k}^{(j)}-\frac{k}{k+1} R_{k-1}^{(j)} r_{0}^{2}
$$

for $k \geq 1$ and $j=0,1$. Then, a method to expand the Hamiltonian (58) expressed in the adapted variables introduced to perform the normal form is the following: to compute the composition of the change (16) adapted to the periodic orbit (that we assume well defined for our concrete orbit, fact that can be tested using the methodology described in Section 2.2), with the Floquet transformation (25) and the complexification (27), and then, to insert this change into the recurrences (59). This method seems to be an efficient way to obtain the desired expansion of the Hamiltonian (58) (see [25], [9], [10], [12] and [28]). We remark that, to compute the changes (16) and (25) we only need to know the explicit expression of the periodic orbit and the variational matrix of the orbit for the initial Hamiltonian (57).

\subsection{Bounds on the norm of the Hamiltonian}

First note that the expansion of the Hamiltonian in (58) is done around $L_{5}$, and not around the periodic orbit. Hence, it is easy to check that this expansion only converges if $r_{0}<1$ (the distance from $L_{5}$ to the primaries, that are the singularities of the Hamiltonian (57)). This implies that, when we introduce the adapted system of coordinates, and we replace $X, Y$ and $Z$ by their expressions in terms of the new coordinates, we need to control the 
value of $r_{0}$ as a function of the allowed range for the new variables, not only to ensure convergence of (58), but also to bound the supremum norm of $H$. The control of this norm, necessary to obtain the estimates for the diffusion time provided by Section 2.8, can be done by looking at the explicit expressions of the adapted coordinates of (16), the Floquet transformation (25), and the complexification (27), and then, computing the norms of the different expansions of the change, as a function of the size of the given domain for the new variables. Then, we can bound the norm of the Hamiltonian using the following lemma.

Lemma 3 Let $A>0$ and let $\left\{P_{k}\right\}_{k \geq 0}$ be a sequence of positive numbers that verify $P_{k+1} \leq \frac{2 k+1}{k+1} P_{k} P_{1}+\frac{k}{k+1} P_{k-1} A$, for $k \geq 1$. We assume that for certain $N$ we have $\left|P_{j}\right| \leq \tilde{P}_{j}$

for $j=1, N-1, N$, and we define $h=\max \left\{\frac{\tilde{P}_{N}}{\tilde{P}_{N-1}}, \tilde{P}_{1}+\sqrt{\tilde{P}_{1}^{2}+A}\right\}$. Then, if $h<1$ we have

$$
\sum_{k \geq N+1} P_{k} \leq \sum_{k \geq N+1} h^{k-N+1} \tilde{P}_{N-1} \leq \tilde{P}_{N-1} \frac{h^{2}}{1-h}
$$

Proof: See [4].

We remark that the recurrence for the $\left\{P_{k}\right\}_{k \geq 0}$ in Lemma 3 is the same obtained taking norms on the $\left\{R_{k}^{(j)}\right\}_{k \geq 0}$ in the recurrence (59). Hence, with this lemma we can bound the norm of the Hamiltonian (using the expansion (58)) as well as the remainder of the expansion when we deal with a finite number of terms.

\subsection{Numerical implementation}

In this section, we describe the algorithm used to perform a computer implementation of the methodology introduced in Section 2 to the case of the RTBP.

Of course, computer assisted works have many inconvenients from a theoretical point of view. First, we have the obvious problem that the arithmetic is not exact, that is, we can only store finite decimal representations for the numerical coefficients, with errors that are propagated with the successive operations. Moreover, we can only deal with truncated expansions for the Taylor and Fourier series. Without losing the formal approach, these problems can be solved, for example, using intervalar arithmetic for the numerical coefficients, and storing for every truncated expansion a bound for the remainder. This methodology allows to do a rigorous computer assisted proof.

If one is only interested in obtaining numerical estimations for the region of effective stability, but based in a rigorous approach, one only needs to look at the most significative terms in the control of this diffusion, ignoring the errors on the computer arithmetic as well as the higher order truncations. Nevertheless, the final result is, if we really work with all the significative terms, "the same" as in the rigorous approach. As we mentioned in the Introduction, this is the approach taken in this paper.

Then, in our software we select certain degree $N$, and we only work with coefficients (that are $2 \pi$-periodic functions on $\theta$ ) of monomials of standard degree less than or equal to $N$. It means that we store for every monomial $q^{l} p^{m} I^{k}$, with $k+|l|_{1}+|m|_{1} \leq N$, a truncated Fourier expansion on $\theta$ for the $2 \pi$-periodic coefficient. For each (complex) Fourier coefficient we store a finite decimal approximation, using the standard double precision of the computer. As it has been mentioned before, we have used this software with $N=16$, and taking the biggest order in the Fourier expansion as 18 . 
When working with finite approximations, we remark that in some cases the Taylor truncations can be done such that they involve terms with order bigger that the one of the normal form, and also, from the analytic character of the Hamiltonian, we have that the coefficients of the Fourier expansions decrease exponentially fast with the order of of the harmonic. So, if we take a "sufficiently" large number of terms, they give the most significative contribution.

With this formulation, we can follow all the theoretical steps of Section 2, bounding (when necessary) the supremum norms (8) and (9) by the norms (10) and (11), evaluated using the truncated expansions.

Hence, after computing a periodic orbit of the Hamiltonian system (57), computation that can be done with high precision, we perform a Fourier analysis of this orbit and of the matrix $B(\theta)$ (see (24)). With these Fourier analysis we compute the Floquet transformation (25), that composed with the complexification (27) allows to compute the function $F$ of (15) expressed in the complexified Floquet variables. Then we solve, up to degree $N$, the equation (14) for $\alpha(\theta, s)$ after substituting $s=F$. This can be done by means of an iterative scheme. It allows to compute a truncate approximation of the canonical change (16) written in the complexified variables (27).

Thus, if we insert this transformation into the Hamiltonian (58), we obtain a Hamiltonian like (30), suitable to compute the normal form. To do this expansion, we use recurrences (59) up to some finite "big" order. We remark that, if we compute a reduced number of terms in these recurrences, we can not ensure that the remainder of this expansion only contains terms of "higher order" of the Taylor expansion around the orbit (we recall that this Taylor expansion is done around $L_{5}$, not around the orbit). This fact implies that, if one wants to justify that working close to the periodic orbit one has small remainder, one needs to take a sufficiently large number of terms in recurrences (59). As it has been mentioned in Section 3.4, we can use Lemma 3 to estimate the error in this truncation. For instance, in this concrete application we have considered the recurrences for $R_{k}^{(0)}$ and $R_{k}^{(1)}$ for $k \leq 30$.

At this point, we have an approximation to the Hamiltonian (expressed in the Floquet complexified variables) given by a polynomial of degree $N$, with $2 \pi$-periodic coefficients on $\theta$. Those coefficients are given by a trigonometric polynomial of certain finite degree. To continue with the computations, we take this expression for the Hamiltonian as "exact" up to degree $N$.

We apply to this Hamiltonian the normal form scheme of Section 2.5. To this end, we choose the formulation explained in Section 2.8, that is, we remove in an increasing form the non-integrable terms of standard degree $3,4, \ldots, N-1$. Then, the final product of these computations is an explicit expression of the normal form up to standard degree $N-1$, and of the generating functions used to put the Hamiltonian in this reduced form. As in the practical implementation we do not take into consideration the errors due to the arithmetic or to the truncated expansions, we take this normal form and the generating functions as correct up to standard degree $N$. Note that the use of standard degree instead of the adapted one forces us to remove some extra monomials. This does not affect the final results since it does not introduce extra small divisors. 


\begin{tabular}{|r|l|}
\hline$x=-4.6699078035506 \mathrm{e}-01$ & $p_{x}=-8.3479753472510 \mathrm{e}-01$ \\
$y=8.6161129973745 \mathrm{e}-01$ & $p_{y}=-4.5245436628470 \mathrm{e}-01$ \\
$z=0.0000000000000 \mathrm{e}+00$ & $p_{z}=2.4999973950378 \mathrm{e}-01$ \\
\hline \multicolumn{2}{|c|}{$T=6.2860040080466 \mathrm{e}+00$} \\
$\alpha_{1}=-1.5906656537706 \mathrm{e}+00$ \\
$\alpha_{2}=2.0827433614129 \mathrm{e}+00$ \\
\hline
\end{tabular}

Table 1: Initial conditions of the chosen periodic orbit. $T$ is the period, and the non-trivial eigenvalues of the monodromy matrix are $\exp \left( \pm \alpha_{j} i\right), j=1,2$.

\subsection{Results in a concrete example}

We start computing the vertical family of periodic orbits for $\mu=0.04$. To do that, we look for fixed points of the return map generated by the Poincaré section $z=0$, and we obtain a curve of fixed points in this hyperplane. Hence, after we pass the stability bifurcation plotted in Figure 1, and as it has been mentioned in Section 3.2.1, we take the orbit with $\dot{z}=0.24999973950378$. The initial conditions, period and normal frequencies of this orbit, are given in Table 1.

Then, we implement the normal form methodology of Section 2 for a generic linearly stable orbit of the RTBP around $L_{5}$, and we particularize the computations on the orbit previously chosen. For this purpose, we work with truncated power series, up to standard degree 16, and with trigonometric polynomials of degree 18 . Hence, after we write the Hamiltonian in the adapted Floquet complexified variables (that is, it takes the form (30)), the normal form is computed up to standard degree 16 as a composition of time one flows associated to generating functions of standard degrees ranging from 2 to 16 .

The following sections are devoted to show some of the results obtained.

\subsubsection{Explicit normal form}

To illustrate the results obtained, we begin giving the first terms of the normal form. To do that, we write this normal form as

$$
\mathcal{N}\left(I_{0}, I_{1}, I_{2}\right)=\sum_{n \in \mathbb{N}^{3}} c_{n} I_{0}^{n_{0}} I_{1}^{n_{1}} I_{2}^{n_{2}}
$$

where $n=\left(n_{0}, n_{1}, n_{2}\right)$, being $I_{0}$ the conjugate action of the angular variable, and $I_{1}, I_{2}$ the actions related to the normal directions. Then, the coefficients $c_{n}$ are displayed in Table 2 up to $|n|_{1} \leq 5$.

The term $c_{0,0,0}$ corresponds to the energy level of the orbit in the RTBP. $c_{1,0,0}$ is the frequency of the periodic orbit, $\omega_{0}=2 \pi / T$, and we recall (see Section 2.3) that $c_{0,1,0}=\alpha_{1} / T, c_{0,0,1}=\alpha_{2} / T$. The coefficient $c_{2,0,0}$ is responsible, at first order, of the variation of the intrinsic frequency of the periodic orbits of the vertical family around the initial one, with respect to the action $I_{0}$. We notice that this coefficient is non-zero, but very small. It implies that this family is close to degenerate, and hence, these orbits are very sensitive to external perturbations (see [28]). 


\begin{tabular}{|c|c|c|c|c|c|c|c|}
\hline$n_{0}$ & $n_{1}$ & $n_{2}$ & $c_{n}$ & $n_{0}$ & $n_{1}$ & $n_{2}$ & $c_{n}$ \\
\hline 0 & 0 & 0 & $3.1711731232826 \mathrm{e}-02$ & 1 & 1 & 2 & $1.4105921461850 \mathrm{e}+10$ \\
\hline 1 & 0 & 0 & $9.9955159098477 e-01$ & 1 & 0 & 3 & $1.9115193141014 \mathrm{e}+10$ \\
\hline 0 & 1 & 0 & $-2.5304878134574 e-01$ & 0 & 4 & 0 & $3.1019518891717 \mathrm{e}+08$ \\
\hline 0 & 0 & 1 & $3.3133026303306 \mathrm{e}-01$ & 0 & 3 & 1 & $3.9719490345200 \mathrm{e}+09$ \\
\hline 2 & 0 & 0 & $-7.0859697825372 e-03$ & 0 & 2 & 2 & $5.1997957166042 e+11$ \\
\hline 1 & 1 & 0 & $2.3478089323297 e+00$ & 0 & 1 & 3 & $1.3724278167916 e+12$ \\
\hline 1 & 0 & 1 & $2.4987325794935 \mathrm{e}+00$ & 0 & 0 & 4 & $9.5691903432773 e+11$ \\
\hline 0 & 2 & 0 & $3.4191968670886 e+01$ & 5 & 0 & 0 & $2.9159565344568 e-04$ \\
\hline 0 & 1 & 1 & 1.79715386 & 4 & 1 & 0 & -3.6 \\
\hline 0 & 0 & 2 & $1.0746686943742 \mathrm{e}+02$ & 4 & 0 & 1 & $-3.6482213649665 e+05$ \\
\hline 3 & 0 & 0 & -2.98185645072 & 3 & 2 & 0 & $-7.7052083427990 e+07$ \\
\hline 2 & 1 & 0 & -7.5 & 3 & 1 & 1 & -3.32 \\
\hline 2 & 0 & 1 & $-7.6071254704866 e+01$ & 3 & 0 & 2 & $1.2824159132491 e+11$ \\
\hline 1 & 2 & 0 & -4.76771251 & 2 & 3 & 0 & $-5.4646830243379 e+09$ \\
\hline 1 & 1 & 1 & -2.14218503906 & 2 & 2 & 1 & $-4.5024287618939 e+10$ \\
\hline 1 & 0 & 2 & $6.7515756251597 e+04$ & 2 & 1 & 2 & $2.7062943714641 \mathrm{e}+13$ \\
\hline 0 & 3 & 0 & -7.46596231110 & 2 & 0 & 3 & $13512343 e+13$ \\
\hline 0 & 2 & 1 & $-6.3172367666144 e+05$ & 1 & 4 & 0 & $-1.6048510841303 e+11$ \\
\hline 0 & 1 & 2 & $4.6984775497921 e+06$ & 1 & 3 & 1 & $-2.0244801390392 e+12$ \\
\hline 0 & 0 & 3 & 5.96452181 & 1 & 2 & 2 & $3267 e+15$ \\
\hline 4 & 0 & 0 & 1.645 & 1 & 1 & 3 & $6.2181555081344 \mathrm{e}+15$ \\
\hline 3 & 1 & 0 & $4.7022354565522 e+03$ & 1 & 0 & 4 & $5.0013972630049 e+15$ \\
\hline 3 & 0 & 1 & $4.7023240428548 \mathrm{e}+03$ & 0 & 5 & 0 & $-1.6820405782961 e+12$ \\
\hline 2 & 2 & 0 & 6.10948694 & 0 & 4 & 1 & $-2.9167436015449 e+13$ \\
\hline 2 & 1 & 1 & $2.6789828013636 \mathrm{e}+06$ & 0 & 3 & 2 & $4.7472462592019 e+16$ \\
\hline 2 & 0 & 2 & $9.7881388815580 e+07$ & 0 & 2 & 3 & $2.2456543810360 e+17$ \\
\hline 1 & 3 & 0 & $2.4411036457491 e+07$ & 0 & 1 & 4 & $3.5758554369748 e+17$ \\
\hline 1 & 2 & 1 & $2.0413328166880 \mathrm{e}+08$ & 0 & 0 & 5 & $1.9045367836910 e+17$ \\
\hline
\end{tabular}

Table 2: Coefficients of the normal form around the chosen orbit up to degree 5. 


\subsubsection{Effective stability estimates}

Next step is to derive a domain where this normal form is convergent. To this end, we take $\mathcal{D}\left(\rho^{(0)}, R^{(0)}\right)$ as initial domain for the complexified Floquet adapted variables (the ones corresponding to Hamiltonian (30)), with $\rho^{(0)}=5 \times 10^{-2}, R_{0}^{(0)}=6.5 \times 10^{-6}$ and $R_{j}^{(0)}=4 \times 10^{-4}, j=1,2,3,4$ (these are suitable values for the following computations). Then, we transform this domain, by means of the complexification (27) and the Floquet change (25), to the variables $(\theta, q, I, p)$ introduced by the canonical transformation (16), and we derive (by bounding the components of the transformations) the upper bounds $R_{0}^{(1)}=9.860 \times 10^{-6}, R_{1}^{(1)}=1.405 \times 10^{-2}, R_{2}^{(1)}=9.681 \times 10^{-3}, R_{3}^{(1)}=6.804 \times 10^{-3}$ and $R_{4}^{(1)}=9.856 \times 10^{-3}$ for the new domain. On this domain, it is not difficult to check that (19) holds, and to obtain the bound $R_{\beta}=2.243700 \times 10^{-2}$ for the expression (20). We note that condition $\mathbf{C}$ "holds" for the chosen orbit, being $A=0.24997269973138$. To be more concrete, working with their truncated expansions, we have that $|\hat{f}|_{\rho^{(0)}} / A$ and $|\hat{g}|_{\rho^{(0)}} / A$ are of order $1.32 \times 10^{-4}$ and $3.94 \times 10^{-4}$ respectively. Then, if we implement numerically the proof of Proposition 1, we obtain, for the current values of $R_{\beta}$ and $\rho^{(0)}$, the estimates $\delta_{0}=0.9534070$ and $\delta_{1}=9.268077 \times 10^{-4}$. As $\delta_{0}>\delta_{1}$, we can ensure that the adapted coordinates introduced by (16) are well defined on $\mathcal{D}\left(\rho^{(0)}, R^{(1)}\right)$. Moreover, we can also obtain (by bounding the components of the transformation (16)) bounds on the size of the initial (complex) domain expressed in the (synodical) coordinates of the RTBP. For instance, we have a bound for $r_{0}$ (the distance to $L_{5}$, see Section 3.3) of order 0.274 (that is smaller than 1). Moreover, if we apply Lemma 3 to these estimates, with $N=30$, we deduce that a bound for the remainder of expansion (58) is $6.82 \times 10^{-17}$. The value $N=30$ has been selected because this is the number of Legendre polynomials taken to perform a numerical implementation of recurrences (59).

Now, we use the method described in Section 2.7 to deduce a domain where we can prove convergence of the normal form transformation up to degrees 10, 12, 14 and 16 . Of course, if we increase the order of the normal form this domain shrinks, but, as we do not find a very strong resonance for these orders, it remains practically constant. It is given by $\mathcal{D}(\rho, R)$, with $\rho=4.038 \times 10^{-2}, R_{0}=6.238 \times 10^{-6}, R_{1}=R_{3}=1.793 \times 10^{-4}$ and $R_{2}=R_{4}=1.349 \times 10^{-4}$. Results on the time needed to leave this domain are plotted in Figure 3. To compute those estimates, we use again Lemma 3 to bound the norm of the Hamiltonian (58) in the considered domain, and we obtain the value $9 \times 10^{-2}$.

It is interesting to compare these results with the stability region obtained using direct numerical integration. Thus, we have taken the Poincaré section $z=0$, and we have selected a mesh of points for the $x$ and $y$ variables. Then, we have used as initial condition for a numerical integration the points given by the $(x, y)$ values of the mesh and the values $\dot{x}, \dot{y}$ and $\dot{z}$ corresponding to the selected periodic orbit (see Table 1 ). If, after 10000 revolutions of the primaries the orbit does not go away, we consider that the initial point is inside the region of stability. The criteria to decide if a point goes away is to check if, at some moment, $y$ becomes negative (this heuristic criteria has been previously used in [16], [30], [10], and see also [27] for a slight modification). The points corresponding to initial conditions of stable orbits have been plotted in Figure 2. Moreover, we have tried to use the normal form computation to determine which points of the mesh above correspond to the effectively stable region. To this end, we have send all the initial conditions through the changes of variables to reach the normal form coordinates (of course, if a point in the mesh is outside the convergence domain of these transformations we assume that it is 

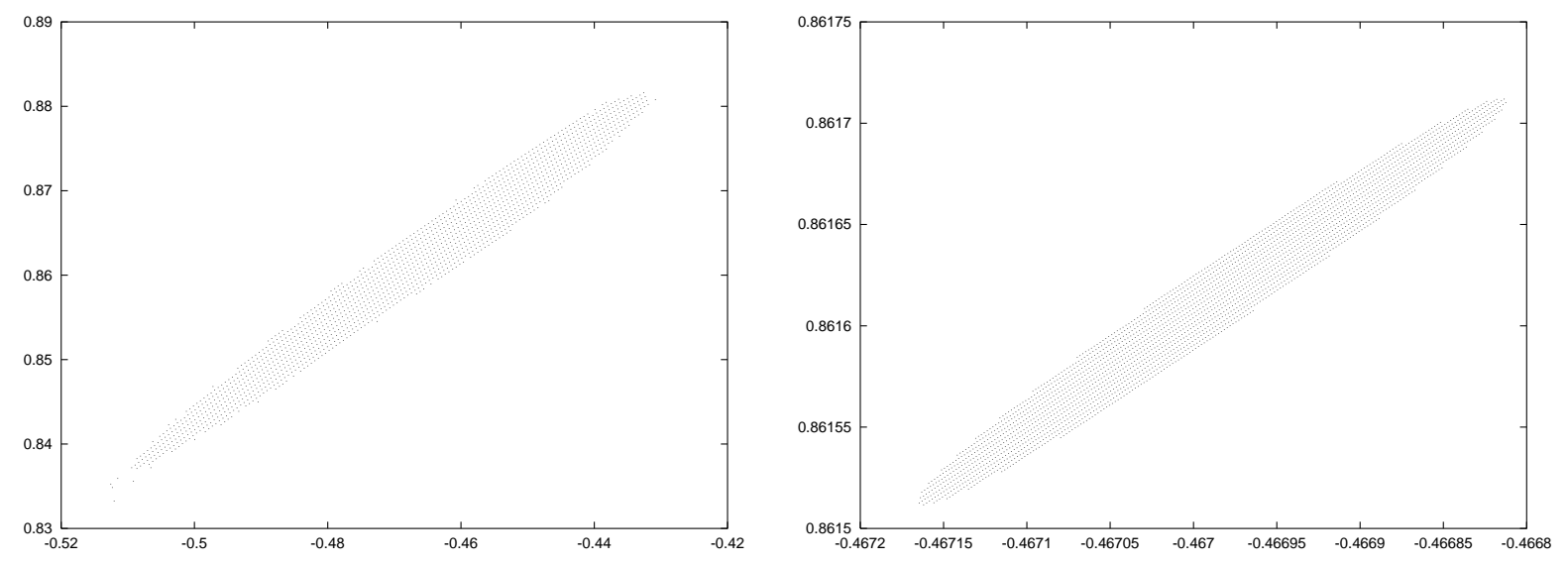

Figure 2: Left: Surviving points of the numerical integration. Right: Points of the left plot for which the coordinates of the normal form up to order 16 belong to $\mathcal{D}(0, R)$.

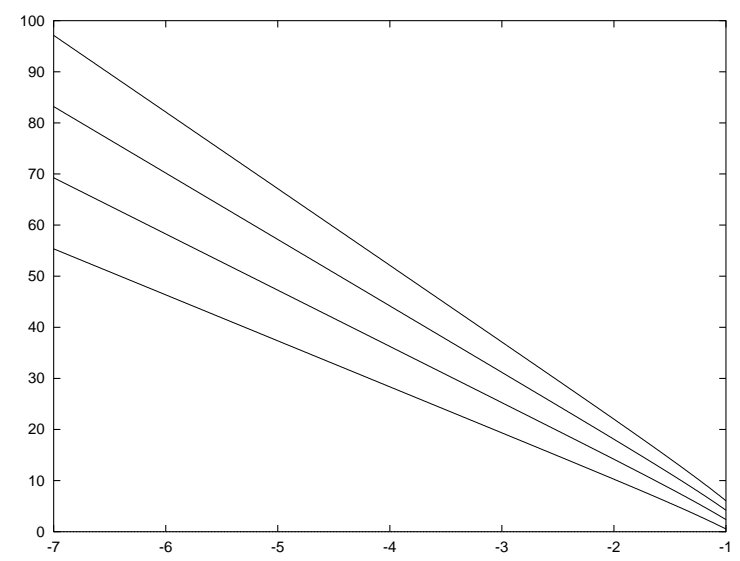

Figure 3: Estimates on the time needed to leave the domain $\mathcal{D}(0, R)$ (see Section 3.6.2). These curves correspond, from bottom to top, to normal forms of orders 10, 12, 14 and 16 , respectively. The horizontal axis is $\log _{10} \delta_{0}$ (see (52)) for the initial condition, and the vertical axis is a lower bound for the time needed to reach $\delta=1$, also in $\log _{10}$ scale.

unstable). Then, it is easy to check if this point is inside the domain of effective stability. So, we have also plotted those points in Figure 2. Note that the region obtained from the normal form computations is about 200 times smaller than the one found by direct numerical simulations. This is due to the local character of normal forms.

\subsubsection{Invariant tori gallery}

Here (Figures 4, 5, 6 and 7) we present some invariant tori of the truncated normal form (see Section 2.6) translated by the different changes of variables, and plotted in the initial coordinates of the RTBP. We also give numerical values for the normal and intrinsic frequencies of the computed tori.

Finally, we explain how to estimate the error on the determination of these tori. If we neglect the errors on the different compositions and of the numerical integrator, we can assume that it is entirely due to the truncated normal form. If we take an initial 

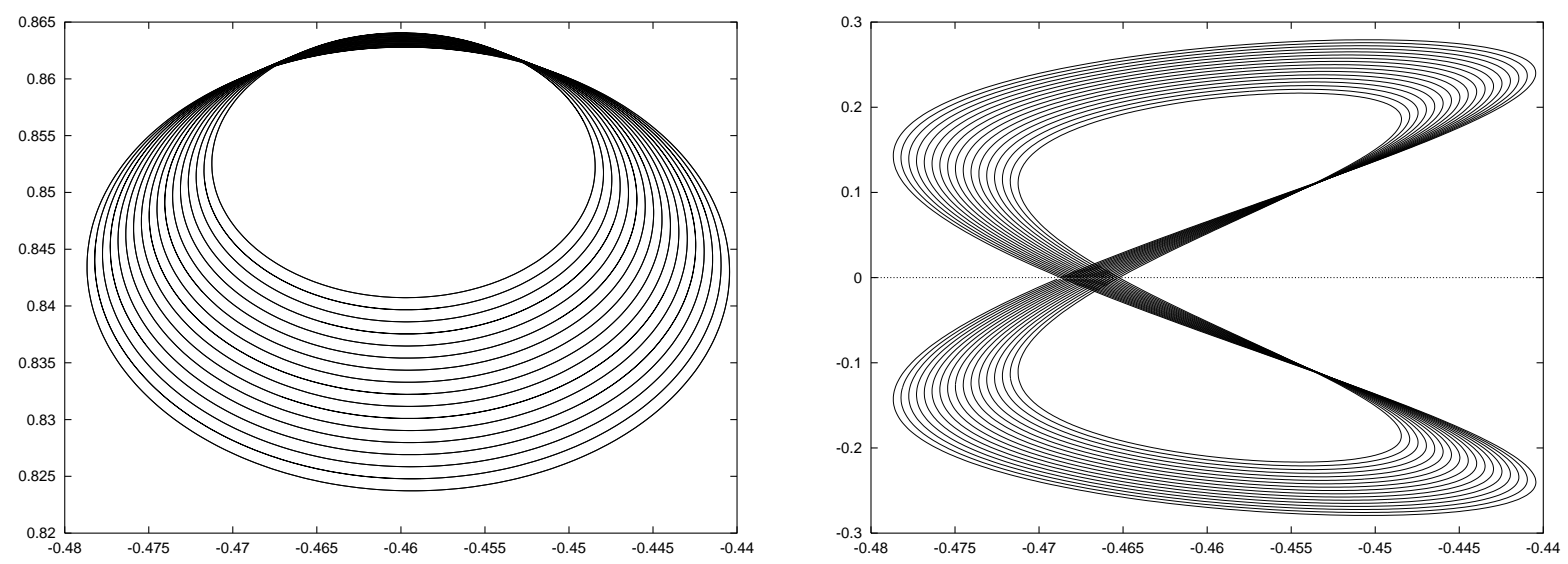

Figure 4: Periodic orbits of the vertical family obtained from the truncated normal form. They can be easily computed by using a standard continuation method, but in the normal form are trivial to obtain putting $I_{1}=I_{2}=0$, and using $I_{0}$ as a parameter in the family. Here, we plot the projections $(x, y)$ (left) and $(x, z)$ (right) of the orbits corresponding to $I_{0}$ from $-8 \times 10^{-3}$ to $8 \times 10^{-3}$ with step $10^{-3}$. We recall that the orbit with $I_{0}=0$ is the initial one.

condition expressed in the coordinates of the truncated normal form, we can explicitly compute the intrinsic frequencies of the corresponding invariant torus, and hence, it can be easily integrated up to time $T_{f}$ by the flow of this truncated normal form. Then, if we send the initial and final points to the corresponding coordinates of the RTBP, and we transform the initial one by the flow of the RTBP up to time $T_{f}$, we can compare both the final points. If there were no error in the determination of the torus, both points should coincide. Their difference is an estimate for the error in the determination of the torus.

Note that if we perform this numerical integration for very long time spans, we will have an extra source of error, coming from the finite precision in the intrinsic frequencies: when we integrate the torus in the normal form coordinates, the product of these frequencies with $T_{f}$ modulus $2 \pi$ is considered. This operation acts as a Bernoulli shift on the significative digits of the frequencies as we increase $T_{f}$, despite of the precision of the initial condition on the torus.

To overcome this problem an alternative method is to compare the actions $I_{0}, I_{1}, I_{2}$ (see (60)). This is done by taking an initial condition in the normal form coordinates, to send it to the RTBP coordinates, to integrate numerically this initial condition, and to send the final point back to the normal form coordinates. Then, we can estimate the error by comparing the values of the actions of the initial and final points (see Figures 5 , 6 and 7$)$.

\subsection{Software}

The software used has been developed by the authors in $\mathrm{C}$ language, and it is specially adapted to the problem. It consist, roughly speaking, in an algebraic manipulator to perform the basic operations (sums, products, Poisson brackets, ... ) for homogeneous polynomials in 5 variables, having as coefficients trigonometric polynomials of some finite (and fixed) order. This strategy improves, in several orders of magnitude, the efficiency (both in speed and memory) obtained by using commercial algebraic manipulators. 

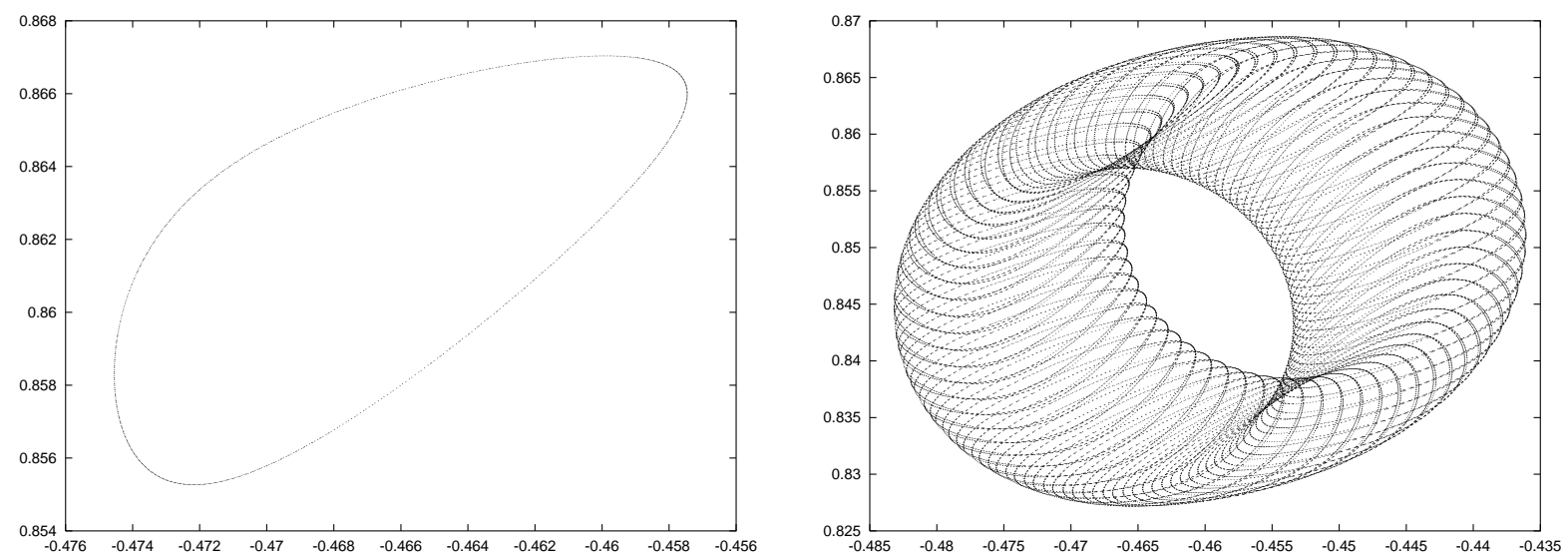

Figure 5: These figures correspond to a 2-D torus near the periodic orbit, given by $I_{0}=I_{1}=0$ and $I_{2}=1 \times 10^{-6}$, and plotted up to time 5000. Left: $(x, y)$ projection of the Poincare section $z=0$ of the torus. Right: $(x, y)$ projection of the same orbit, plotting a point every 0.1 units of time. The intrinsic frequencies of this torus (with the determination given by the truncated normal form) are $\omega_{0}=0.9995541833$ and $\omega_{2}=$ 0.3315682317. The normal one is $\omega_{1}=-0.2528624981$. The error in the determination of this torus (see Section 3.6.3) computed by comparing points in the RTBP is (up to time $10000)$ of order $1 \times 10^{-6}$. The errors on the actions $I_{0}, I_{1}$ and $I_{2}$, up to the same time, are of order $1.5 \times 10^{-12}, 1.2 \times 10^{-20}$ and $3.4 \times 10^{-12}$ respectively.
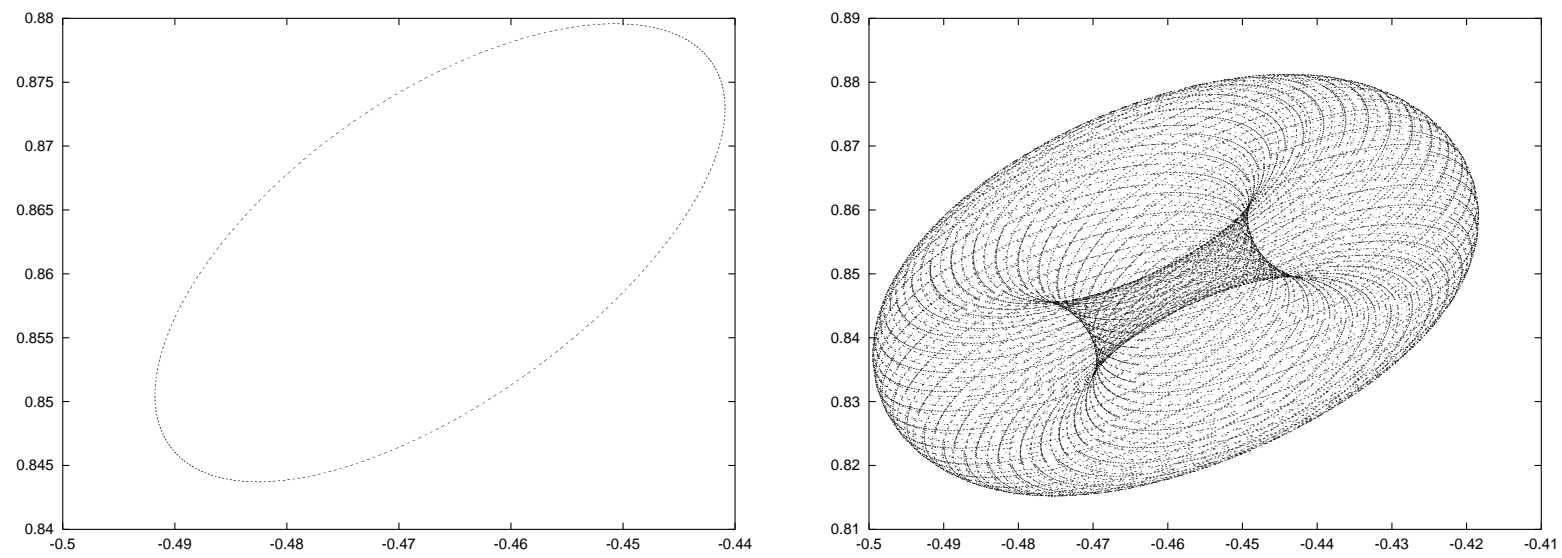

Figure 6: Like Figure 5, but for a torus in the other family around the initial orbit: $I_{0}=$ $I_{2}=0$, and $I_{1}=1 \times 10^{-5}$. The intrinsic frequencies of this torus are $\omega_{0}=0.9995746152$ and $\omega_{1}=-0.2523861773$, and the normal one is $\omega_{2}=0.3330679465$. The errors for this torus are of order $2 \times 10^{-6}$ (in the RTBP), $3.9 \times 10^{-10}$ (for $I_{0}$ ), $2.2 \times 10^{-11}$ (for $I_{1}$ ) and $7.7 \times 10^{-14}\left(\right.$ for $\left.I_{2}\right)$. 

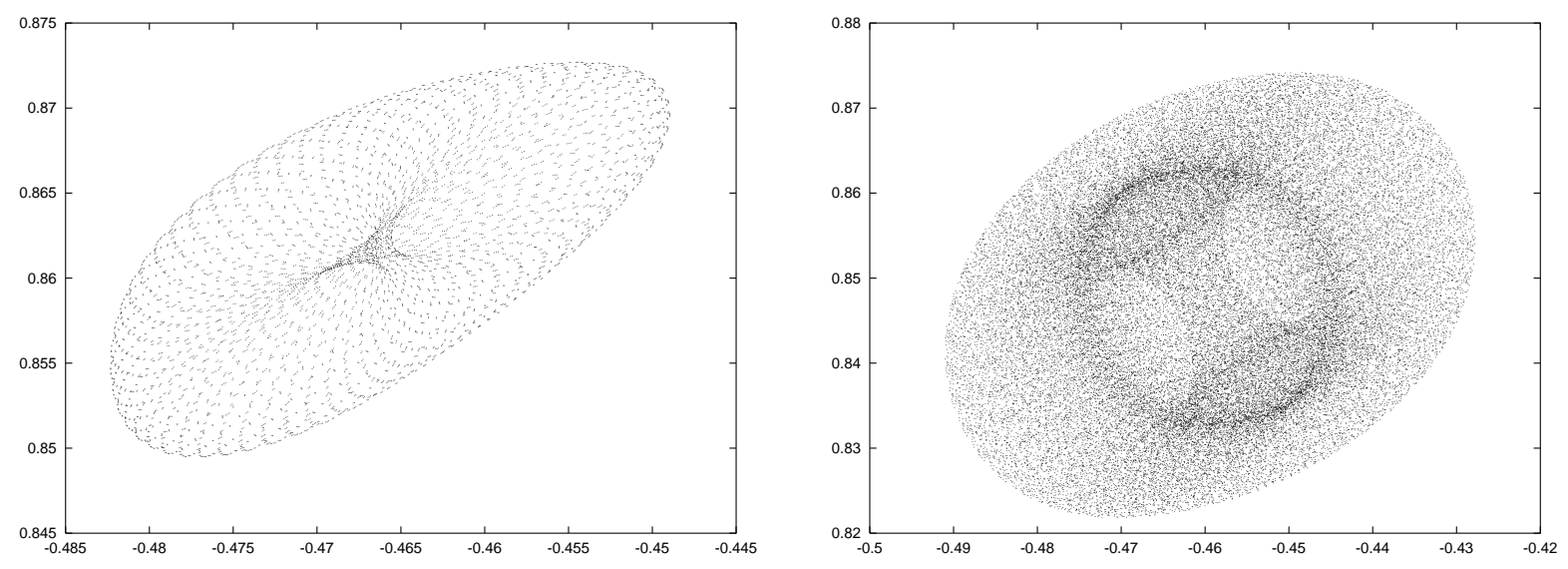

Figure 7: Like Figure 5, but now for a 3-D torus. It is obtained putting $I_{0}=0$ and $I_{1}=I_{2}=1 \times 10^{-6}$. The left plot is obtained integrating up to time 40000 . The right one up to time 5000 (a point is plotted every 0.1 units of time ). The intrinsic frequencies of this torus are $\omega_{0}=0.9995565341, \omega_{1}=-0.2527934779$ and $\omega_{2}=0.3317656274$. The errors for this torus (up to time 20000) are of order $1.7 \times 10^{-5}$ (in the RTBP), $3.7 \times 10^{-12}$ (for $\left.I_{0}\right), 3.7 \times 10^{-12}\left(\right.$ for $\left.I_{1}\right)$ and $2.8 \times 10^{-11}\left(\right.$ for $\left.I_{2}\right)$.

\section{Proof of Proposition 1}

In this section we prove Proposition 1 (see Section 2.2), used to bound the domain of definition of the adapted system of coordinates introduced by (16).

To this end, to work with the transformation (18) we introduce

$$
\begin{aligned}
& F(\theta, \beta)=\hat{g}(\theta)+i \hat{f}(\theta)+\left(\hat{f}^{\prime}(\theta)-i \hat{g}^{\prime}(\theta)\right) \beta \equiv F_{0}(\theta)+F_{1}(\theta) \beta, \\
& G(\theta, \beta)=\hat{g}(\theta)-i \hat{f}(\theta)+\left(\hat{f}^{\prime}(\theta)+i \hat{g}^{\prime}(\theta)\right) \beta \equiv G_{0}(\theta)+G_{1}(\theta) \beta .
\end{aligned}
$$

With these notations, we can write the relations

$$
\begin{aligned}
& y+i x=A \exp (i \theta)(1+\beta)+F(\theta, \beta), \\
& y-i x=A \exp (-i \theta)(1+\beta)+G(\theta, \beta) .
\end{aligned}
$$

Before contining the proof, let us introduce some additional notations. To deal with the different $2 \pi$-periodic functions on $\theta$, we introduce a new variable $\zeta=\exp (i \theta)$, that overcomes the difficulty of the identification modulus $2 \pi$ of the variable $\theta$. Hence, given $h(\theta)$, a $2 \pi$-periodic analytic function on $\theta$, we have that the Fourier expansion of $h$,

$$
h(\theta)=\sum_{k \in \mathbb{Z}} h_{k} \exp (i k \theta),
$$

becomes in this new variable a Laurent expansion,

$$
\hbar(\zeta)=\sum_{k \in \mathbb{Z}} h_{k} \zeta^{k}
$$

Moreover, if $h$ converges on a complex strip of width $\rho$, then $\hbar$ converges on the complex annulus $\mathcal{A}\left(R_{\zeta}\right) \equiv\left\{\zeta \in \mathbb{C}:\left(R_{\zeta}\right)^{-1} \leq|\zeta| \leq R_{\zeta}\right\}$, with $R_{\zeta}=\exp (\rho)$. To work with this Laurent expansion, we introduce the norm:

$$
|\hbar|_{R}=\sum_{k \in \mathbb{Z}}\left|h_{k}\right| R^{|k|}
$$


The notation $|.|_{R}$ for this norm can be confusing, because is the same one used for the norm $|.|_{\rho}$ defined in (10), but we remark that clearly $|\hbar|_{R_{\zeta}}=|h|_{\rho}$. For further uses of this norm, we note its multiplicative character: $\left|\hbar^{(1)} \hbar^{(0)}\right|_{R} \leq\left|\hbar^{(1)}\right|_{R}\left|\hbar^{(0)}\right|_{R}$. Moreover, we have the following bounds related to the $|\cdot|_{R}$ norm:

Lemma 4 With the notations given above, and for any $\zeta_{1}, \zeta_{2} \in \mathcal{A}(R), R>1$, we have:

(i) $\left|\hbar\left(\zeta_{2}\right)-\hbar\left(\zeta_{1}\right)\right| \leq\left|\hbar^{\prime}\right|_{R}\left|\zeta_{2}-\zeta_{1}\right|$.

$(i i)\left|\hbar\left(\zeta_{2}\right)-\hbar\left(\zeta_{1}\right)-\hbar^{\prime}\left(\zeta_{1}\right)\left(\zeta_{2}-\zeta_{1}\right)\right| \leq \frac{1}{2}\left|\hbar^{\prime \prime}\right|_{R}\left|\zeta_{2}-\zeta_{1}\right|^{2}$.

\section{Proof:}

(i) We take $k \in \mathbb{Z} \backslash\{0\}$. If $k>0$ :

$$
\zeta_{2}^{k}-\zeta_{1}^{k}=\left(\zeta_{2}-\zeta_{1}\right)\left(\zeta_{2}^{k-1}+\zeta_{2}^{k-2} \zeta_{1}+\cdots+\zeta_{1}^{k-1}\right)
$$

and hence, $\left|\zeta_{2}^{k}-\zeta_{1}^{k}\right| \leq k R^{k-1}\left|\zeta_{2}-\zeta_{1}\right|$. Analogously, if $k<0$ :

$$
\begin{aligned}
\zeta_{2}^{k}-\zeta_{1}^{k} & =\zeta_{2}^{k} \zeta_{1}^{k}\left(\zeta_{1}^{-k}-\zeta_{2}^{-k}\right)=\zeta_{2}^{k} \zeta_{1}^{k}\left(\zeta_{1}-\zeta_{2}\right)\left(\zeta_{1}^{-k-1}+\zeta_{1}^{-k-2} \zeta_{2}+\cdots+\zeta_{2}^{-k-1}\right)= \\
& =\left(\zeta_{1}-\zeta_{2}\right)\left(\zeta_{2}^{k} \zeta_{1}^{-1}+\zeta_{2}^{k+1} \zeta_{1}^{-2}+\cdots+\zeta_{2}^{-1} \zeta_{1}^{-k}\right)
\end{aligned}
$$

and then we have $\left|\zeta_{2}^{k}-\zeta_{1}^{k}\right| \leq|k| R^{|k|+1}\left|\zeta_{2}-\zeta_{1}\right|$. From here the result follows immediately.

(ii) In this case, for $k>1$, we have

$$
\begin{aligned}
\zeta_{2}^{k} & -\zeta_{1}^{k}-k \zeta_{1}^{k-1}\left(\zeta_{2}-\zeta_{1}\right)=\zeta_{2}^{k}-k \zeta_{1}^{k-1} \zeta_{2}+(k-1) \zeta_{1}^{k}= \\
& =\left(\zeta_{2}-\zeta_{1}\right)\left(\zeta_{2}^{k-1}+\zeta_{2}^{k-2} \zeta_{1}+\cdots+\zeta_{2} \zeta_{1}^{k-2}+(1-k) \zeta_{1}^{k-1}\right)= \\
& =\left(\zeta_{2}-\zeta_{1}\right)^{2}\left(\zeta_{2}^{k-2}+2 \zeta_{2}^{k-3} \zeta_{1}+\cdots+(k-1) \zeta_{1}^{k-2}\right),
\end{aligned}
$$

and then, $\left|\zeta_{2}^{k}-\zeta_{1}^{k}-k \zeta_{1}^{k-1}\left(\zeta_{2}-\zeta_{1}\right)\right| \leq(1+2+\cdots+(k-1)) R^{k-2}=\frac{(k-1) k}{2} R^{k-2}$. If $k<0$, we have

$$
\begin{aligned}
& \zeta_{2}^{k}-\zeta_{1}^{k}-k \zeta_{1}^{k-1}\left(\zeta_{2}-\zeta_{1}\right)=\zeta_{1}^{k-1} \zeta_{2}^{k}\left(\zeta_{1}^{-k+1}-k \zeta_{2}^{-k+1}+(k-1) \zeta_{1} \zeta_{2}^{-k}\right)= \\
& \quad=\zeta_{1}^{k-1} \zeta_{2}^{k}\left(\zeta_{1}-\zeta_{2}\right)\left(\zeta_{1}^{-k}+\zeta_{2} \zeta_{1}^{-k-1}+\cdots+\zeta_{2}^{-k-1} \zeta_{1}+k \zeta_{2}^{-k}\right)= \\
& \quad=\zeta_{1}^{k-1} \zeta_{2}^{k}\left(\zeta_{1}-\zeta_{2}\right)^{2}\left(\zeta_{1}^{-k-1}+2 \zeta_{2} \zeta_{1}^{-k-2}+\cdots+(-k) \zeta_{2}^{-k-1}\right)= \\
& \quad=\left(\zeta_{1}-\zeta_{2}\right)^{2}\left(\zeta_{1}^{-2} \zeta_{2}^{k}+2 \zeta_{1}^{-3} \zeta_{2}^{k+1}+\cdots+(-k) \zeta_{1}^{k-1} \zeta_{2}^{-1}\right)
\end{aligned}
$$

and then, $\left|\zeta_{2}^{k}-\zeta_{1}^{k}-k \zeta_{1}^{k-1}\left(\zeta_{2}-\zeta_{1}\right)\right| \leq \frac{(-k)(-k+1)}{2} R^{|k|+2}\left|\zeta_{1}-\zeta_{2}\right|^{2}=\frac{k(k-1)}{2} R^{|k|+2}\left|\zeta_{1}-\zeta_{2}\right|^{2}$. Hence, the bound is proved.

To use this new notation, we introduce the function $\hat{F}(\zeta, \beta)$ by the identity $\hat{F}(\zeta, \beta) \equiv$ $F(\theta, \beta)$. similarly, we define $\hat{F}_{0}, \hat{F}_{1}, \hat{G}, \hat{G}_{0}$ and $\hat{G}_{1}$ as a functions of $\zeta$ and $\beta$.

Then, to study the injectivity of (18), we take points $\left(\zeta_{0}, \beta_{0}\right)$ and $\left(\zeta_{1}, \beta_{1}\right)$ for which we assume we have the same image for $x$ and $y$ by the action of the transformation

$$
(\zeta, \beta) \mapsto(x, y)
$$


induced by (18). We will prove that, if $\hat{f}$ and $\hat{g}$ are small enough, this is only possible if $\left(\zeta_{0}, \beta_{0}\right)=\left(\zeta_{1}, \beta_{1}\right)$, at least if we take complex values for $\zeta$ and $\beta$ close enough to $|\zeta|=1$ and $\beta=0$. To check that, we deduce from (61) the equalities

$$
\begin{aligned}
A \zeta_{0}\left(1+\beta_{0}\right)+\hat{F}\left(\zeta_{0}, \beta_{0}\right) & =A \zeta_{1}\left(1+\beta_{1}\right)+\hat{F}\left(\zeta_{1}, \beta_{1}\right) \\
A \zeta_{0}^{-1}\left(1+\beta_{0}\right)+\hat{G}\left(\zeta_{0}, \beta_{0}\right) & =A \zeta_{1}^{-1}\left(1+\beta_{1}\right)+\hat{G}\left(\zeta_{1}, \beta_{1}\right)
\end{aligned}
$$

and from here, we have

$$
\begin{gathered}
A\left(1+\beta_{0}\right)\left(\zeta_{0}-\zeta_{1}\right)+A \zeta_{0}\left(\beta_{0}-\beta_{1}\right)=A\left(\zeta_{1}-\zeta_{0}\right)\left(\beta_{1}-\beta_{0}\right)+\hat{F}_{0}\left(\zeta_{1}\right)-\hat{F}_{0}\left(\zeta_{0}\right)+\hat{F}_{1}\left(\zeta_{1}\right) \beta_{1}-\hat{F}_{1}\left(\zeta_{0}\right) \beta_{0}, \\
A\left(1+\beta_{0}\right)\left(\zeta_{1}-\zeta_{0}\right)+A \zeta_{0}\left(\beta_{0}-\beta_{1}\right)=\zeta_{0} \zeta_{1}\left(\hat{G}_{0}\left(\zeta_{1}\right)-\hat{G}_{0}\left(\zeta_{0}\right)+\hat{G}_{1}\left(\zeta_{1}\right) \beta_{1}-\hat{G}_{1}\left(\zeta_{0}\right) \beta_{0}\right),
\end{gathered}
$$

expressions that can be written in the following form:

$$
\begin{aligned}
& \left(\begin{array}{cc}
A\left(1+\beta_{0}\right) & A \zeta_{0} \\
-A\left(1+\beta_{0}\right) & A \zeta_{0}
\end{array}\right)\left(\begin{array}{c}
\zeta_{0}-\zeta_{1} \\
\beta_{0}-\beta_{1}
\end{array}\right)= \\
= & \left(\begin{array}{c}
\hat{F}_{0}^{\prime}\left(\zeta_{0}\right)\left(\zeta_{1}-\zeta_{0}\right)+\hat{F}_{1}^{\prime}\left(\zeta_{0}\right) \beta_{0}\left(\zeta_{1}-\zeta_{0}\right)+\hat{F}_{1}\left(\zeta_{0}\right)\left(\beta_{1}-\beta_{0}\right) \\
\zeta_{0}^{2} \hat{G}_{0}^{\prime}\left(\zeta_{0}\right)\left(\zeta_{1}-\zeta_{0}\right)+\zeta_{0}^{2}\left(\hat{G}_{1}\left(\zeta_{0}\right)\left(\beta_{1}-\beta_{0}\right)+\hat{G}_{1}^{\prime}\left(\zeta_{0}\right)\left(\zeta_{1}-\zeta_{0}\right) \beta_{0}\right)
\end{array}\right)+ \\
& +\left(\begin{array}{c}
\hat{R}_{\hat{F}}+A\left(\beta_{1}-\beta_{0}\right)\left(\zeta_{1}-\zeta_{0}\right) \\
\hat{R}_{\hat{G}}
\end{array}\right),
\end{aligned}
$$

being

$$
\begin{aligned}
\hat{R}_{\hat{F}}= & \hat{F}_{0}\left(\zeta_{1}\right)-\hat{F}_{0}\left(\zeta_{0}\right)-\hat{F}_{0}^{\prime}\left(\zeta_{0}\right)\left(\zeta_{1}-\zeta_{0}\right)+\left(\hat{F}_{1}\left(\zeta_{1}\right)-\hat{F}_{1}\left(\zeta_{0}\right)-\hat{F}_{1}^{\prime}\left(\zeta_{0}\right)\left(\zeta_{1}-\zeta_{0}\right)\right) \beta_{0}+ \\
& +\left(\hat{F}_{1}\left(\zeta_{1}\right)-\hat{F}_{1}\left(\zeta_{0}\right)\right)\left(\beta_{1}-\beta_{0}\right)
\end{aligned}
$$

and

$$
\begin{aligned}
\hat{R}_{\hat{G}}= & \zeta_{0}\left(\zeta_{1}-\zeta_{0}\right)^{2} \hat{G}_{0}^{\prime}\left(\zeta_{0}\right)+\zeta_{0}\left(\zeta_{1}-\zeta_{0}\right)\left(\hat{G}_{1}\left(\zeta_{1}\right)\left(\beta_{1}-\beta_{0}\right)+\hat{G}_{1}^{\prime}\left(\zeta_{0}\right)\left(\zeta_{1}-\zeta_{0}\right) \beta_{0}\right)+ \\
& +\zeta_{0}^{2}\left(\hat{G}_{1}\left(\zeta_{1}\right)-\hat{G}_{1}\left(\zeta_{0}\right)\right)\left(\beta_{1}-\beta_{0}\right)+\zeta_{0} \zeta_{1}\left(\hat{G}_{0}\left(\zeta_{1}\right)-\hat{G}_{0}\left(\zeta_{0}\right)-\hat{G}_{0}^{\prime}\left(\zeta_{0}\right)\left(\zeta_{1}-\zeta_{0}\right)\right)+ \\
& +\zeta_{0} \zeta_{1}\left(\hat{G}_{1}\left(\zeta_{1}\right)-\hat{G}_{1}\left(\zeta_{0}\right)-\hat{G}_{1}^{\prime}\left(\zeta_{0}\right)\left(\zeta_{1}-\zeta_{0}\right)\right) \beta_{0} .
\end{aligned}
$$

From (64), we obtain

$$
\begin{aligned}
& \left(\begin{array}{l}
\zeta_{0}-\zeta_{1} \\
\beta_{0}-\beta_{1}
\end{array}\right)= \\
= & \left(\begin{array}{ll}
\frac{1}{2 A\left(1+\beta_{0}\right)} & -\frac{1}{2 A\left(1+\beta_{0}\right)} \\
\frac{1}{2 A \zeta_{0}} & \frac{1}{2 A \zeta_{0}}
\end{array}\right)\left(\begin{array}{ll}
\hat{F}_{0}^{\prime}\left(\zeta_{0}\right)+\hat{F}_{1}^{\prime}\left(\zeta_{0}\right) \beta_{0} & \hat{F}_{1}\left(\zeta_{0}\right) \\
\zeta_{0}^{2} \hat{G}_{0}^{\prime}\left(\zeta_{0}\right)+\zeta_{0}^{2} \hat{G}_{1}^{\prime}\left(\zeta_{0}\right) \beta_{0} & \zeta_{0}^{2} \hat{G}_{1}\left(\zeta_{0}\right)
\end{array}\right)\left(\begin{array}{c}
\zeta_{1}-\zeta_{0} \\
\beta_{1}-\beta_{0}
\end{array}\right)+ \\
& +\left(\begin{array}{ll}
\frac{1}{2 A\left(1+\beta_{0}\right)} & -\frac{1}{2 A\left(1+\beta_{0}\right)} \\
\frac{1}{2 A \zeta_{0}} & \frac{1}{2 A \zeta_{0}}
\end{array}\right)\left(\begin{array}{c}
\hat{R}_{\hat{F}}+A\left(\beta_{1}-\beta_{0}\right)\left(\zeta_{1}-\zeta_{0}\right) \\
\hat{R}_{\hat{G}}
\end{array}\right) \equiv \\
\equiv & M\left(\begin{array}{c}
\zeta_{1}-\zeta_{0} \\
\beta_{1}-\beta_{0}
\end{array}\right)+N .
\end{aligned}
$$

At this point, we proceed taking the domain for $\zeta$ and $\beta$ given in the statement of the proposition, where we expect the transformation (62) to be injective. In what follows we describe how to test if this assumption holds. We recall that in this statement we are 
assuming $\hat{f}$ and $\hat{g}$ well defined in a complex strip of width $\rho$, for certain $\rho>0$, that is, if $|\operatorname{Im}(\theta)| \leq \rho$. In consequence, $\hat{F}_{0}, \hat{G}_{0}, \hat{F}_{1}$ and $\hat{G}_{1}$ are analytic on $\mathcal{A}\left(R_{\zeta}\right)$. With respect to $\beta$, we consider complex values with $|\beta| \leq R_{\beta}$. Then, we want to check the injectivity of the transformation in the domain generated by fixed values of $\delta$ and $R_{\beta}$.

For this purpose, we use Lemma 4 to bound the expressions $\hat{R}_{\hat{F}}$ and $\hat{R}_{\hat{G}}$ of (65) and (66) in the annulus $\mathcal{A}\left(R_{\zeta}\right)$. This can be done bounding the $|\cdot|_{R_{\zeta}}$ norm of the $\zeta$-functions $\hat{F}_{0}, \hat{F}_{0}^{\prime}$, $\hat{F}_{0}^{\prime \prime}, \hat{G}_{0}, \hat{G}_{0}^{\prime}$ and $\hat{G}_{0}^{\prime \prime}$. Then, we obtain bounds for the components of $N, N^{\top}=\left(N_{1}, N_{2}\right)$, of the form:

$$
\left|N_{j}\right| \leq N_{j, 0}\left|\zeta_{1}-\zeta_{0}\right|^{2}+2 N_{j, 1}\left|\zeta_{1}-\zeta_{0}\right|\left|\beta_{1}-\beta_{0}\right|, j=1,2
$$

that holds if $\zeta_{0}, \zeta_{1} \in \mathcal{A}\left(R_{\zeta}\right)$ and $\left|\beta_{0}\right|,\left|\beta_{1}\right| \leq R_{\beta}$. Using these estimates, we obtain from $(67)$

$$
\begin{aligned}
& \left|\zeta_{0}-\zeta_{1}\right|+\left|\beta_{0}-\beta_{1}\right| \leq \\
& \quad \leq \frac{1}{1-M_{*}}\left(\left(N_{1,0}+N_{2,0}\right)\left|\zeta_{1}-\zeta_{0}\right|^{2}+2\left(N_{1,1}+N_{2,1}\right)\left|\zeta_{1}-\zeta_{0}\right|\left|\beta_{1}-\beta_{0}\right|\right),
\end{aligned}
$$

where $M_{*}$, that we assume verifies $M_{*}<1$, is a bound (in the considered domain) for the matrix norm of $M$ induced by the vectorial norm $|\cdot|_{1}$ of $\mathbb{C}^{2}$. Then, for the admissible values of $\left(\zeta_{j}, \beta_{j}\right), j=0,1$, for which (68) holds, we have that either $\left(\zeta_{0}, \beta_{0}\right)=\left(\zeta_{1}, \beta_{1}\right)$ or

$$
1 \leq \frac{\left|\zeta_{1}-\zeta_{0}\right|}{1-M_{*}} \max \left\{N_{1,0}+N_{2,0}, 2\left(N_{1,1}+N_{2,1}\right)\right\}
$$

From (69) we deduce the local injectivity of (62). The maximum value allowed for $\left|\zeta_{1}-\zeta_{0}\right|$ defines $\delta_{0}\left(R_{\beta}, \rho\right)$ on the statement of Proposition 1. To deduce global injectivity, we write (63) in the following form

$$
\begin{aligned}
& \left(\begin{array}{l}
\zeta_{0}-\zeta_{1}-\frac{1}{2\left(1+\beta_{0}\right)}\left(\beta_{1}-\beta_{0}\right)\left(\zeta_{1}-\zeta_{0}\right) \\
\beta_{0}-\beta_{1}-\frac{1}{2 \zeta_{0}}\left(\beta_{1}-\beta_{0}\right)\left(\zeta_{1}-\zeta_{0}\right)
\end{array}\right)= \\
= & \left(\begin{array}{ll}
\frac{1}{2 A\left(1+\beta_{0}\right)} & -\frac{1}{2 A\left(1+\beta_{0}\right)} \\
\frac{1}{2 A \zeta_{0}} & \frac{1}{2 A \zeta_{0}}
\end{array}\right)\left(\begin{array}{c}
\hat{F}\left(\zeta_{1}, \beta_{1}\right)-\hat{F}\left(\zeta_{0}, \beta_{0}\right) \\
\zeta_{0} \zeta_{1}\left(\hat{G}\left(\zeta_{1}, \beta_{1}\right)-\hat{G}\left(\zeta_{0}, \beta_{0}\right)\right)
\end{array}\right) \equiv S .
\end{aligned}
$$

If the components of $S$ are bounded by $S_{1}$ and $S_{2}$ when $\zeta_{0}, \zeta_{1} \in \mathcal{A}\left(R_{\zeta}\right)$ and $\left|\beta_{0}\right|,\left|\beta_{1}\right| \leq R_{\beta}$, then we have from $(70)$ :

$$
\begin{gathered}
\left|\zeta_{0}-\zeta_{1}\right|\left(1-\frac{1}{2\left(1-R_{\beta}\right)}\left|\beta_{1}-\beta_{0}\right|\right) \leq S_{1}, \\
\left|\beta_{1}-\beta_{0}\right|\left(1-\frac{R_{\zeta}}{2}\left|\zeta_{1}-\zeta_{0}\right|\right) \leq S_{2}
\end{gathered}
$$

From that, we deduce that the pairs $\left(\zeta_{0}, \beta_{0}\right)$ and $\left(\zeta_{1}, \beta_{1}\right)$ in the considered domain, with the same image by (62), are necessarily close. As we have $\left|\beta_{1}-\beta_{0}\right| \leq 2 R_{\beta}$, we deduce from (71) that it is necessary to take

$$
\left|\zeta_{0}-\zeta_{1}\right| \leq \frac{S_{1}}{1-\frac{R_{\beta}}{1-R_{\beta}}} \equiv \delta_{1}\left(R_{\beta}, \rho\right),
$$

where if $R_{\beta}<1 / 2$, we have $\delta_{1}>0$. Assuming $\delta_{1}$ smaller than the value of $\delta_{0}$ that guarantee local injectivity in (69), we have global injectivity for $\zeta \in \mathcal{A}\left(R_{\zeta}\right)$ and $|\beta| \leq R_{\beta}$, that is, in terms of $\theta$, if $|\operatorname{Im}(\theta)| \leq \rho$. 


\section{Acknowledgements}

The authors want to thank interesting remarks from Carles Simó. The research of À. Jorba has been supported by the Spanish grant DGICYT PB94-0215, the EC grant ERBCHRXCT940460, the Catalan grant CIRIT 1996SGR-00105. The research of J. Villanueva has been supported by the UPC grant PR9409.

\section{References}

[1] Arnol'd V. I.: Proof of a Theorem of A. N. Kolmogorov on the Persistence of QuasiPeriodic Motions under Small Perturbations of the Hamiltonian, Russ. Math. Surveys, 18:5 (1963), pp. 9-36.

[2] Arnol'd V. I.: Instability of Dynamical Systems with Several Degrees of Freedom, Sov. Math. Dokl. 5:3 (1964), pp. 581-585.

[3] Broer H. W., Huitema G. B. and Sevryuk M. B.: Quasi-Periodic Motions in Families of Dynamical Systems: Order amidst Chaos, Lecture Notes in Math., Vol. 1645, Springer 1996.

[4] Celletti A. and Giorgilli A.: On the Stability of the Lagrangian Points in the Spatial Restricted Problem of Three Bodies, Cel. Mech., 50 (1991), pp. 31-58.

[5] Delshams A. and Gutiérrez P.: Effective Stability and KAM Theory, J. Diff. Eq., 128 (1996), pp. 415-490.

[6] Delshams A. and Gutiérrez P.: Estimates on Invariant Tori Near an Elliptic Equilibrium Point of a Hamiltonian System, J. Diff. Eq., 131 (1996), pp. 277-303.

[7] Eliasson L. H.: Perturbations of Stable Invariant Tori for Hamiltonian Systems, Ann. Sc. Norm. Super. Pisa, Cl. Sci., Ser. IV, 15:1 (1988), pp. 115-147.

[8] Giorgilli A., Delshams A., Fontich E., Galgani L. and Simó C.: Effective Stability for a Hamiltonian System Near an Elliptic Equilibrium Point, with an Application to the Restricted Three Body Problem, J. Diff. Eq. 77 (1989), pp. 167-198.

[9] Gómez G., Jorba A., Masdemont J. and Simó C.: A Quasiperiodic Solution as a Substitute of L4 in the Earth-Moon System, Proceedings of the 3rd International Symposiun on Spacecraft Flight Dynamics, ESA Publications Division, ESTEC, Noordwijk, Holland (1991), pp. 35-41.

[10] Gómez G., Jorba A., Masdemont J. and Simó C.: Study of Poincaré Maps for Orbits Near Lagrangian Points, ESOC contract 9711/91/D/IM(SC), Final Report (1993).

[11] Jorba A. and Masdemont J.: Nonlinear Dynamics in an Extended Neighbourhood of the Translunar Equilibrium Point, Hamiltonian Systems with Three or More Degrees of Freedom, C. Simó (Ed.), NATO Adv. Sci. Inst. Ser. C Math. Phys. Sci. Kluwer Acad. Publ. Dordrecht, Holland. To appear in 1997. 
[12] Jorba A. and Simó C.: Effective Stability for Periodically Perturbed Hamiltonian Systems, Hamiltonian Mechanics, Integrability and Chaotic Behaviour, J. Seimenis (Ed.), vol. 331 of NATO Adv. Sci. Inst. Ser. B Phys. Plenum Press, New York (1994), pp. $245-252$.

[13] Jorba A. and Villanueva J.: Effective Stability Around Periodic Orbits of the Spatial RTBP, Hamiltonian Systems with Three or More Degrees of Freedom, C. Simó (Ed.), NATO Adv. Sci. Inst. Ser. C Math. Phys. Sci. Kluwer Acad. Publ. Dordrecht, Holland. To appear in 1997.

[14] Jorba A. and Villanueva J.: On the Normal Behaviour of Partially Elliptic Lower Dimensional Tori of Hamiltonian Systems, to appear in Nonlinearity.

[15] Lochak P. and Neishtadt A.: Estimates in the Theorem of N. N. Nekhoroshev for Systems with a Quasi-Convex Hamiltonian, Chaos 2 (1992), pp. 495-499.

[16] McKenzie R. and Szebehely V.: Non-Linear Stability Motion Around the Triangular Libration Points, Cel. Mech. 23 (1981), pp. 223-229.

[17] Morbidelli A. and Giorgilli A.: Superexponential Stability of KAM Tori, J. Statist. Phys. 78 (1995), pp. 1607-1617.

[18] Nekhoroshev N. N.: An Exponential Estimate of the Time of Stability of NearlyIntegrable Hamiltonian Systems, Russ. Math. Surveys, 32:6 (1977), pp. 1-65.

[19] Niederman L.: Stability over Exponentially Long Times in the Planetary Problem, Nonlinearity 9 (1996), pp. 1703-1751.

[20] Perry A. D. and Wiggins S.: KAM Tori are Very Sticky: Rigorous Lower Bounds on the Time to Move Away from an Invariant Lagrangian Torus with Linear Flow, Physica D 71 (1994), pp. 102-121.

[21] Pöschel J.: Integrability of Hamiltonian Systems on Cantor Sets, Comm. Pure Appl. Math., 35 (1982), pp. 653-695.

[22] Pöschel J.: Nekhoroshev Estimates for Quasi-Convex Hamiltonian Systems, Math. Z., 213 (1993), pp. 187-217.

[23] Sevryuk M.: Excitation of Elliptic Normal Modes of Invariant Tori in Hamiltonian Systems. Preprint (1997).

[24] Siegel C. and Moser J. K.: Lectures on Celestial Mechanics, Springer-Verlang, Berlin, Heidelberg, New York (1971).

[25] Simó C.: Estabilitat de Sistemes Hamiltonians, Memorias de la Real Academia de Ciencias y Artes de Barcelona, 48 (1989), pp. 303-348.

[26] Simó C.: Averaging Under Fast Quasiperiodic Forcing, Hamiltonian Mechanics, Integrability and Chaotic Behaviour, J. Seimenis (Ed.), vol. 331 of NATO Adv. Sci. Inst. Ser. B Phys. Plenum Press, New York (1994), pp. 13-34. 
[27] Simó C.: Effective Computations in Hamiltonian Dynamics, in Cent ans après les Méthodes Nouvelles de H. Poincaré, Société Mathématique de France (1996), pp. $1-23$.

[28] Simó C., Gómez G., Jorba A. and Masdemont J.: The Bicircular Model Near the Triangular Libration Points of the RTBP, From Newton to Chaos, A.E. Roy and B.A. Stevens (Eds.), Plenum Press, New York (1995), pp. 343-370.

[29] Szebehely V.: Theory of Orbits, Academic Press (1967).

[30] Szebehely V. and Premkumar R.: Global Sensitivity to Velocity Errors at the Libration Points, Cel. Mech. 28 (1982), pp. 195-200. 